Published in final edited form as:

Nature. 2021 March ; 591(7848): 124-130. doi:10.1038/s41586-021-03234-7.

\title{
Global Absence and Targeting of Protective Immune States in Severe COVID-19
}

\author{
Alexis J. Combes ${ }^{1,2,3, \ddagger,{ }^{*}, \text { Tristan Courau }}{ }^{1,2,3, \ddagger}$, Nicholas F. Kuhn ${ }^{1,2, \ddagger}$, Kenneth H. Hu ${ }^{1,2, \ddagger}$, \\ Arja Ray ${ }^{1,2, \ddagger}$, William S. Chen ${ }^{2,4}$, Nayvin W. Chew ${ }^{1,2,3}$, Simon J. Cleary ${ }^{2,5}$, Divyashree \\ Kushnoor $^{1,2,3}$, Gabriella C. Reeder ${ }^{1,2,3}$, Alan Shen ${ }^{1,2,3}$, Jessica Tsui ${ }^{1,2,3}$, Kamir J. Hiam- \\ Galvez $^{2,6,7}$, Priscila Muñoz-Sandoval2,7,8, Wandi S Zhu' ${ }^{2,7,8}$, David S. Lee ${ }^{2,9}$, Yang Sun ${ }^{2,9}$, \\ Ran You ${ }^{1,2}$, Mélia Magnen ${ }^{2,5}$, Lauren Rodriguez ${ }^{2,6}$, K.W. Im ${ }^{1,2,3}$, Nina K. Serwas ${ }^{1,2}$, \\ Aleksandra Leligdowicz ${ }^{5}$, Colin R. Zamecnik ${ }^{2,10}$, Rita P. Loudermilk ${ }^{2,10}$, Michael R. \\ Wilson $^{2,10}$, Chun J. Ye ${ }^{2,9}$, Gabriela K. Fragiadakis ${ }^{2,3,9}$, Mark R. Looney ${ }^{2,5}$, Vincent Chan ${ }^{1,2}$, \\ Alyssa Ward ${ }^{9}$, Sidney Carrillo ${ }^{5}$, The UCSF COMET Consortium, Michael Matthay ${ }^{5}$, David \\ J. Erle ${ }^{2,3,5}$, Prescott G. Woodruff ${ }^{2,5}$, Charles Langelier ${ }^{11}$, Kirsten Kangelaris ${ }^{12}$, Carolyn M.

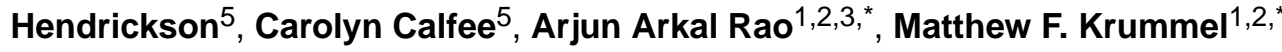 \\ 1 Department of Pathology, San Francisco, 513 Parnassus Ave, HSW512, San Francisco, CA \\ 94143-0511, USA \\ 2ImmunoX Initiative, San Francisco, 513 Parnassus Ave, HSW512, San Francisco, CA \\ 94143-0511, USA \\ 3UCSF CoLabs, San Francisco, 513 Parnassus Ave, HSW512, San Francisco, CA 94143-0511, \\ USA \\ ${ }^{4}$ Department of Radiation Oncology, San Francisco, 513 Parnassus Ave, HSW512, San \\ Francisco, CA 94143-0511, USA \\ ${ }^{5}$ Division of Pulmonary and Critical Care Medicine, Department of Medicine and the \\ Cardiovascular Research Institute, San Francisco, 513 Parnassus Ave, HSW512, San Francisco, \\ CA 94143-0511, USA
}

\footnotetext{
”To whom correspondence should be addressed. Alexis.Combes@ucsf.edu, ArjunArkal.Rao@ucsf.edu, Matthew.Krummel@ucsf.edu. ₹These authors contributed equally Author Contributions

AJC, TC, NFK, KHH, AR, AAR, WSC, NW,SC, MFK performed and provided supervision of experiments, generated and analyzed data, and contributed to the manuscript by providing figures and tables. AJC, TC, NFK, KHH, AR, AAR, MFK had full access to all of the data in the study and take responsibility for the integrity of the data and the accuracy of the analyses. AJC, TC, NFK, KHH, AR, AAR, WSC, performed computational analyses of the data. AJC, TC, KHH, VC, NWC, DK, GCR, AS, JT, KJHG, PMS, WSZ, DSL, YS, were part of the early morning COVID-19 processing team who performed the whole blood single cell sequencing. The UCSF COMET Consortium included all other scientist involved in the generation of data included in this study. CL provided viral load obtained from nasal swab of COVID-19 patient. CJY, ML and SC provided blood from healthy donors and helped with PBMC preparation. NKS, KWI, RY, MM,LR, AL, CRZ, RPL performed assays of SARS infection and serum antibodies and cytokines. MW and MM oversaw studies of serum cytokines and antibodies from the COVID-19 patient cohort and provided intellectual input. PGW, DJE, GKF, CC, CL, CH, MFK are leadership members of the COMET study and were actively involved in the establishment of the pipeline and patient consent and the direction of projects. PGW, $\mathrm{CC}, \mathrm{CH}, \mathrm{AW}$ and $\mathrm{SC}$ actively participated in patient and control cohort enrollment. AJC, TC, NFK, KHH, AR, AAR, WSC, MFK wrote the manuscript. AJC, AAR and MFK supervised the project. All authors edited and critically revised the manuscript for important intellectual content and gave final approval for the version to be published.

Conflict of interest Statement

The authors declare no competing financial interests.

Supplementary Information and Method

Detailed material and method and supplementary table describing patient cohort can be found in the supplementary information file
} 
${ }^{6}$ Departments of Otolaryngology, San Francisco, 513 Parnassus Ave, HSW512, San Francisco, CA 94143-0511, USA

${ }^{7}$ Department of Microbiology \& Immunology, San Francisco, 513 Parnassus Ave, HSW512, San Francisco, CA 94143-0511, USA

${ }^{8}$ Sandler Asthma Basic Research Center, San Francisco, 513 Parnassus Ave, HSW512, San Francisco, CA 94143-0511, USA

Institute of Human Genetics and Division of Rheumatology, Department of Medicine, San Francisco, 513 Parnassus Ave, HSW512, San Francisco, CA 94143-0511, USA

${ }^{10}$ Weill Institute for Neurosciences, Department of Neurology, San Francisco, 513 Parnassus Ave, HSW512, San Francisco, CA 94143-0511, USA

${ }^{11}$ Division of Infectious Disease, Department of Medicine, San Francisco, 513 Parnassus Ave, HSW512, San Francisco, CA 94143-0511, USA

12 Division of Hospital Medicine, San Francisco, 513 Parnassus Ave, HSW512, San Francisco, CA 94143-0511, USA

\section{Abstract}

While SARS-CoV-2 infection has pleiotropic and systemic effects in some patients ${ }^{1-3}$, many others experience milder symptoms. We sought a holistic understanding of the severe/mild distinction in COVID-19 pathology, and its origins. We performed a whole-blood preserving single-cell analysis protocol to integrate contributions from all major cell types including neutrophils, monocytes, platelets, lymphocytes and the contents of serum. Patients with mild COVID-19 disease display a coordinated pattern of interferon-stimulated gene (ISG) expression ${ }^{3}$ across every cell population and these cells are systemically absent in patients with severe disease. Severe COVID-19 patients also paradoxically produce very high anti-SARS-CoV-2 antibody titers and have lower viral load as compared to mild disease. Examination of the serum from severe patients demonstrates that they uniquely produce antibodies that functionally block the production of the mild disease-associated ISG-expressing cells, by engaging conserved signaling circuits that dampen cellular responses to interferons. Overzealous antibody responses pit the immune system against itself in many COVID-19 patients and perhaps in other viral infections and this study defines targets for immunotherapies in severe patients to re-engage viral defense.

\section{One Sentence Summary:}

In severe COVID-19 patients, the immune system fails to generate cells that define mild disease; antibodies in their serum actively prevents the successful production of those cells.

\section{Global Loss of Interferon Signatures in Severe COVID-19}

To understand immune biology amongst COVID-19 patients, we compared them to patients presenting with similar respiratory symptoms but who were not infected with the SARSCoV-2 virus. We enrolled 21 SARS-CoV-2 positive inpatients, 11 inpatients with similar clinical presentations consistent with acute lung injury (ALI) or acute respiratory distress syndrome (ARDS), who were SARS-CoV-2 negative-those caused by other infections or 
of unknown origin — and 14 control individuals. We further categorized these individuals as 'mild/moderate' (M/M: short stays in hospital with no need for mechanical ventilation and intensive care) or 'severe' (requiring intubation and intensive care) according to the full clinical course of their disease (Fig 1a/ED1a and Table S1). Hence, our study includes patients with mild/moderate $(n=11)$ or severe $(n=10)$ COVID-19 and patients with mild/moderate $(n=6)$ or severe $(n=5)$ non-COVID-19 ALI/ARDS. With the exception of one individual, all our patients who presented with mild/moderate disease remained mild/ moderate during hospitalization (ED1a), suggesting that mild/moderate and severe are more stable states rather than transient phases of disease in this cohort.

Since the majority of COVID-19 mortality is among patients with ARDS—characterized by an exuberant immune response with prominent contributions from neutrophils, monocytes, platelets-we focused upon collecting these cells along with other major populations. We thus performed single-cell RNA sequencing (scRNA-seq) on RBC-depleted blood samples from all individuals. After merging, batch-correction and doublet-removal, our data comprised 116,517 cells (Fig 1b/ED1b) among which we identified neutrophils, platelets, mononuclear phagocytes, T/NK cells, B cells, plasma cells and eosinophils (Fig 1b/ED1c). We confirmed a positive association between neutrophil frequency and disease severity and an inverse correlation for lymphoid populations (Fig 1b/ED1d) (1-3). At this level of resolution, findings were similar between SARS-CoV-2 negative and positive individuals (ED1f-e.)

Within the neutrophils, we identified seven subtypes (Fig 1c/ED2a), consistent with previous studies $(2,4)$. One population, harboring a strong interferon-stimulated gene (ISG) signature and henceforth termed ISG neutrophils, was highly enriched in SARS-CoV-2 positive patients but not in those whose disease was severe (Fig 1d-e/ED2b). Separate pseudotime analysis (ED2d-g) placed the ISG subtype as a late-stage of differentiation and was the only such state found significantly altered between mild/moderate and severe patients (ED2e) and specifically within the SARS-CoV-2 positive individuals (Fig 1f/ED2c). ISG signature genes include master anti-viral regulators such as ISG15 and IFITM3 which restricts viral entry into the cytosol (5).

We also analyzed differentially expressed genes (DEG) from SARS-CoV-2 positive versus negative patients, and from mild/moderate versus severe patients across all neutrophils. ISG signature genes were expressed differentially higher in all neutrophil subsets, specifically in SARS-CoV-2 positive mild/moderate patients, as compared to SARS-CoV-2 positive severe patients (Fig 1g/ED2h-n). In contrast, a separate neutrophil degranulation gene program is upregulated in neutrophils from mild/moderate patients as compared to severe regardless of COVID status (ED2o-p). This suggests a shared program of degranulation enhancement in all respiratory infections regardless of causative pathogen, and a global induction of the ISG program in all neutrophils in mild/moderate SARS-CoV-2 positive patients that is absent in severe ones (3).

Assessing the mononuclear phagocytes-monocytes, macrophages, dendritic cells and plasmacytoid dendritic cells (pDC)—yielded 7 clusters of transcriptionally distinct cells subsets, evenly distributed across our cohort (ED3a-f). We identified an ISG expressing 
classical monocytes cluster as being enriched in SARS-CoV-2 positive patients, and particularly those having mild/moderate disease, similarly to neutrophils (Fig 2a/ED4a-c). ISG monocytes also expressed genes associated with glycolysis, compared to a S100A12expressing subset that were enriched for genes associated with oxidative phosphorylation, consistent with previous reports in bacterial sepsis (6) (ED4d). DEG analysis demonstrated that ISGs were the dominant genes associated with mild/moderate phenotypes when the entire mononuclear phagocyte pool was assessed (ED4e).

ISG monocytes and ISG neutrophils frequencies were strongly correlated with one another in mild/moderate SARS-CoV-2 positive individuals (Fig 2b/ED4f). A comprehensive analysis of $\mathrm{T}$ cell and $\mathrm{B}$ cell frequencies (ED5) demonstrated that both cell types were also significantly enriched in ISG signatures, specifically in mild/moderate COVID-19 patients (Fig 2c). The frequencies of ISG+ cells in one compartment correlated with the frequency of ISG expressing cells in another, for example ISG+ T cells and ISG+ neutrophils, uniquely in mild/moderate patients (ED4g). Spearman correlation analysis across multiple cell types in all patients thus showed a collection of correlated ISG+ populations and a second anticorrelated block of other cell populations, notably those expressing S100A12 (Fig 2d).

Our scRNA-seq whole blood dataset also allowed us to identify platelets and subset them based on established platelet signature genes (Fig 1a/ED1d). Analysis of these found six clusters, including three ("H3F3B", "HIST1H2AC", and "RGS18") still carrying transcripts acquired from parental cells, megakaryocytes (ED6a-b) (7). "HIST1H2AC" subset was only modestly depleted in severe COVID-19 patients suggesting a skewing away from 'younger' cells (ED6c). This was supported when overlaying the expression of BCL2L1 onto our dataset, which has been identified as a 'molecular clock' for platelet lifetime (8). This identified a histone-rich H3F3B cluster as representing 'young' platelets (ED6d), a result supported by a second signature of transcripts in young, reticulated platelets (9) (ED6e). Pseudotime analysis rooted at this H3F3B (ED6f-g) suggested again that platelets from all patients with disease were broadly overrepresented at the end of the trajectory (ED6h). While we did not identify a distinct ISG+ cluster (ED6i), akin to myeloid and lymphoid cells, ISG signature scores in platelets from mild/moderate patients was increased relative to severe patients, particularly for SARS-CoV-2 infected patients (Fig 2e).

Platelet scRNA-seq also permitted the identification of heterotypic aggregates between platelets and non-platelets by using a 'Platelet First' approach (ED7a-c). This approach revealed the presence of platelet transcripts associated with cells that also bore signatures of other major blood cell types (ED7a-c). We found no profound differences in frequencies of cell types in this 'Platelet First' object compared to the original data set (ED7e). This suggests that, at least in circulating blood, platelets form aggregates indiscriminately with varying other cell types without favoring one or the other.

\section{Holistic Assessment of Severe COVID-19}

After observing that ISG expression profiles were elevated in every cell type among patients with mild/moderate disease but globally reduced with severe illness, we turned to a holistic view of disease states. Phenotypic earth mover's distance (PhEMD) (10) embedding of 
patients based on their subtype frequencies revealed eight distinct groups of patients (Fig 2f/ED7f) wherein progression from A through $\mathrm{H}$ represent patients with generally increasing relative frequency of neutrophils. Intermediates C, D, G and $\mathrm{H}$ include patients with relative enrichment in monocytes and $\mathrm{E}$ represents patients with an enrichment of ISG neutrophils and mostly consists of SARS-CoV-2 positive patients with mild/moderate disease (Fig $2 \mathrm{~g}-$ h). In contrast, Group G, which is an alternative and 'severe' fate for patients is highly enriched for neutrophils and has a dominance of S100A12 versus ISG neutrophils (ED7f).

Examination of serum IFNa levels could not explain this loss of ISG+ cell populations in severe patients since severe patients were found with substantial IFNa production (Fig 3a). However, ISG populations were strongly correlated with low severity of COVID-19 illness, with serum IFNa concentration and lower plasma levels of SP-D (indicative of alveolar epithelial injury) (ED8a). When compared to a high-dimensional panel of plasma protein levels (ED8c), most ISG subtypes clustered together and correlated with factors indicative of a strong ISG and Th1 response (CXCL1/6/10/11, TNFB, IL-12B, MCP-2/4). An unexpected anticorrelate of the ISG state was the concentration of serum antibodies against the SARS-CoV-2 Spike and Nucleocapsid proteins (Fig 3b/ED8a).

This anticorrelation was profound and not strongly mirrored in higher total levels of $\operatorname{IgG}$ antibodies or immune complexes in severe patient sera (ED8d-f). We considered it a paradox that severe patients have higher levels of potentially neutralizing antibodies. This is in apparent contradiction with a previous report showing that viral load is associated with severity and mortality in COVID-19 $(11,12)$, a difference which could be explained by the fact that these studies compare amongst patients with high mortality, which was a very rare event in our cohort (Sup Table S1). At day of admission, both antibody specificities were anticorrelated with the viral load as assessed from nasal swabs (Fig 3c/ ED8b) consistent with though not definitive for being neutralizing. As increased antibody titers and decreased viral load have been reported to be a feature of later disease stage (13), we considered the hypothesis that our observed mild/moderate disease simply preceded severe disease. However, antibody titers in severe patients are consistently higher compared to mild/moderate patients over time, even two weeks beyond symptom onset (Fig 3d/ED8e), and only one of our $19 \mathrm{mild} /$ moderate patients would go on to exhibit a severe disease (ED1b). Finally, we observed no statistical correlation between days of onset and the presence of ISG+ cell populations (ED8a). These elements would seem to argue against a simple temporal relationship between mild/moderate and severe states and led us to investigate a systemic etiology for this split in states in serum.

\section{COVID-19 Serum Antibodies Antagonize Interferon Responses}

Considering this enhancement of antibodies, we first asked whether serum from severe patients also contained antibodies against ISG-expressing cells by directly applying serum to peripheral blood mononuclear cells (PBMCs from heathy individuals) cultured with and without IFNa (ED9a-d). We observed serum IgG binding from 2 mild/moderate and 2 severe COVID-19 patients (ED9a). However, staining was highly variable on different cell types (ED9b-c), both with and without prior IFNa stimulation, suggesting that patients may each have unique combinations of specificities. For instance, examining patient 1050 whose 
serum did not stain ISG-differentiated cells directly, we found evidence of antibodies to IFNa (right inset Fig 3e), consistent with a very recent study (14) that also found these in approximately $12 \%$ of COVID patients. This patient was unique in our cohort and IFNa reactivity further does not explain the majority of severe patients lacking ISG cells.

We separately tested whether factors in the serum of severe patients affect the induction of the ISG signature gene pattern, using IFITM3 as a marker, in response to culture with IFNa. We thus mixed patient serum at 5\% into an IFNa stimulation of healthy PBMCs and found that, whereas control serum or serum from mild/moderate patients had no effect on differentiation as measured by either IFITM3 level or the frequencies of CD14+CD16 intermediate monocytes produced, all severe patient serum tested had profound effects, varying from complete block to partial inhibition. (Fig 3e/g and S9d-e).

To test if antibodies in severe patient serum were responsible for this inhibition of IFNa response, we pre-adsorbed patients' sera with Protein A/G beads to deplete them. This relieved the block in both IFITM3 induction and the total yield of interferon-stimulated monocytes (Fig $3 \mathrm{f}-\mathrm{g}$ ). A similar block and release through antibody-absorbtion was observed for IFNa-dependent ISG signature generation in other populations including lymphocytes (Fig 3h/ED9f). We consider it likely, since profound IFN responses are dependent on a positive feedback loop from initial Interferon a Receptor (IFNAR) signaling (15), that IFN response in lymphocytes benefits from IFNAR signaling amplification in monocytes. We also confirmed an inhibition of ISG cell population generation by severe serum in a second validation cohort composed of $8 \mathrm{M} / \mathrm{M}$ and 6 severe patients (ED10a, table S2).

\section{Severe COVID-19 Patients Antagonize IFNAR Signaling Through $\mathrm{FC}_{\boldsymbol{\gamma}} \mathrm{RIIb}$}

Probing the mechanism for this result, we found that blocking antibodies to Fc Receptors (CD16/CD64/CD32) during culture with IFNa and patient serum restored IFITM3 induction in cells cultured with serum from severe patient both in discovery (Fig 4a/e left) and validation (ED10b) cohorts. Fc receptor blocking restored not only IFITM3 induction but other ISG's, such as IFI27, ISG15 and MX1 (ED10c). These results and the absence of augmented cell death in PBMC cultured with serum from severe patient (ED10d) suggested that antibodies present in serum from severe patients trigger $\mathrm{Fc}$ receptor signaling, which inhibits transcriptional responses following IFNAR engagement.

We considered that such a mechanism might represent a fundamental way for antibody generation to downregulate an interferon cascade and therefore we tested whether $\mathrm{Fc}$ receptor activation via cross linking antagonized IFITM3 induction by IFNa. PBMC subjected to individual crosslinking of CD32, but not CD16 or CD64, demonstrated dramatically less IFITM3 induction (Fig 4b-c/ED10e) while crosslinking of all FcR together induced pro-inflammatory cytokine production (ED10f).

Returning to severe COVID-19 serum effects, we found that blocking CD32 alone restored IFITM3 induction in PBMC's cultured with IFNa in the presence of severe serum (Fig 4d-e right). Previous studies demonstrated that Fc $\gamma$ RIIb (CD32b) blockade could lead to IFN-like 
responses in dendritic cells and monocytes (16) while binding of the activating Fc receptor Fc $\gamma$ RIIa (CD32a) elicit viral immunity (17). Consistent with those previous studies, we found that blocking of FC $\gamma \mathrm{RIIb}$, but not FC $\gamma \mathrm{RIIa}$, rescued IFITM3 induction in monocytes cultured with serum from severe patients (ED10g).

Taken together, inhibition of a phenotype of ISG-expressing immune populations in severe patients correspond to antagonism of IFNAR signaling via $\mathrm{FC} \gamma \mathrm{RIIb}$ receptor signaling by their antibodies. In our cohort, this general antibody-mediated effect manifests in almost all severe patients, whereas antibodies against the cytokine IFNa itself were seen only in one of seven patients, and those antibodies blocked ISG function but not via FcRs (Fig 4a). With regard to specificity, it is notable that very recent works have highlighted autoantibodies in COVID-19 binding to targets as diverse as phospholipids (18) and endothelial proteins (19) but that not all patients had developed each specificity. Our work likewise found antibody binding to mixtures of immune cells themselves and it is possible that, in the course of an infection, incomplete tolerance in the B cell compartment may include recognition of a great many host proteins including those on immune cells. While it will be important to study the likely diverse nature of antibody specificities in COVID-19, afucosylation of antibodies, which modifies selectivity for FcR subtypes, as well as differential IgG subclass selectivity is also emerging as a distinguishing feature (20) and we speculate that variable levels of these IgG subclasses in sera combined with varying affinities for different $\mathrm{Fc}$ receptors could result in stronger signaling through inhibitory FC $\gamma \mathrm{RIIb}$. Further work will be necessary to characterize the relative contributions of these IgG subclasses and their specificities. Regardless, our study suggests that this global targeting of ISG archetypes might be addressable with drugs such as rituximab to reduce B cell responses (21) perhaps in the presence of convalescent serum, through introduction of IVIG to compete with serum antibodies for FcR engagement (22), or with rapid development of antibodies that clinically block FC $\gamma$ RIIb.

\section{Material and Methods}

\section{Patients, participants, severity score, and clinical data collection:}

Patients admitted to the Hospital of the University of California with known or presumptive COVID-19 were screened within 3 days of hospitalization. Patients, or a designated surrogate, provided informed consent to participate in the study. This study includes a subset of patient enrolled between April 8 and May 1 in the COMET (COVID-19 Multiimmunophenotyping projects for Effective Therapies; https://www.comet-study.org/) study at UCSF. COMET is a prospective study that aims to describe the relationship between specific immunologic assessments and the clinical courses of COVID-19 in hospitalized patients. Healthy donors (Ctrl) were adults with no prior diagnosis of or recent symptoms consistent with COVID-19. This analysis includes samples from participants who provided informed consent directly, via a surrogate, or otherwise in accordance with protocols approved by the regional ethical research boards and the Declaration of Helsinki. For inpatients, clinical data were abstracted from the electronic medical record into standardized case report forms. We used both a severity score at the time of sampling and at the end of hospitalization (ED1a). In both cases, severity assessment was based on three main 
parameters: level of care, need for mechanical ventilation, and time under mechanical ventilation. Mild/moderate patients are floor/ICU patients who did not require mechanical ventilation during their time of hospitalization and spent no more than 1 day in ICU. Severe patients are patients who required intensive care and mechanical ventilation (typically 5 days or more). Therefore, our validation cohort is composed of 21 COVID-19 positive patients (11 mild/moderate and 10 severe), 11 COVID-19 negative patient (6 mild/moderate and 5 severe), and 14 Healthy participants. We also collected and used serum from a validation cohort composed of 14 SARS-CoV-2 positives. Samples were collected and severity was assessed as previously described for initial cohort. This discovery cohort is composed of $8 \mathrm{mild} /$ moderate and 6 severe patients. Information on age, sex, type of infection, days of on onset, viral load, and CBC count are listed in Table S1. The study is approved by the Institutional Review board: IRB\# 20-30497.

\section{Isolation of blood cells and processing for scRNA-seq:}

ScRNA-seq was performed on fresh whole blood in order to preserve granulocytes. Briefly, peripheral blood was collected into EDTA tubes (BD, catalog no. 366643). Whole blood was prepared by treatment of $500 \mu \mathrm{L}$ of peripheral blood with $\mathrm{RBC}$ lysis buffer (Roche, 11-814-389-001) according to manufacturer's procedures. Cells were then counted and 15.000 cells per individual were directly loaded in the Chromium ${ }^{\mathrm{TM}}$ Controller for partitioning single cells into nanoliter-scale Gel Bead-In-Emulsions (GEMs) following manufacturer's procedures (10x genomics). Some samples were pooled together (at 15,000 cells/ sample) prior to GEM partitioning. Single Cell 5' reagent kit v5.1 was used for reverse transcription, cDNA amplification and library construction of the gene expression libraries (10x Genomics) following the detailed protocol provided by 10x Genomics. Libraries were sequenced on an Illumina NovaSeq6000 using 28 cycles for R1 and 98 cycles for R2. All samples were encapsulated, and cDNA was generated within 6 hours after blood draw.

\section{PBMC co-culture experiment with patient serum and flow cytometry analysis:}

PBMCs were isolated from EDTA-anticoagulated whole blood from healthy donors using Polymorphprep (Alere Technologies), and resuspended in culture medium (RPMI $1640+$ $10 \%$ FBS). For detection of neutralization of interferon stimulation, autologous serum or clinical study participant sera $(10 \mu \mathrm{l})$ were plated with IFNa (Stemcell IFN alpha-2A; final concentration of $1 \mathrm{pg} / \mathrm{ul}$ ) in a total volume of $200 \mu \mathrm{l}$ before addition of $2.5 \times 10^{5}$ PBMCs. After incubation for 24 hours, PBMCs were assayed for IFNa-induced IFITM3 upregulation and CD14/CD16 levels and fractions by flow cytometry. After surface staining and addition of fixable live/dead violet dye (ThermoFisher; \#L34955), intracellular detection of IFITM3 was done using the eBioscience Foxp3 / Transcription Factor Staining Buffer Set (ThermoFisher; \#00-5523-00) and following the manufacturer's instructions. For, FcR blocking experiments, $\mathrm{Fc}$ receptors were blocked with unconjugated anti-CD16 (clone 3G8; BioLegend; \#302002), anti-CD32 (clone FUN-2; BioLegend; \#303202), anti-CD64 (clone 10.1; BioLegend; \#305002), anti-CD32a(Clone IV.4,BioXcell) and anti-CD32b/c (clone S18005H Biolegend) with 0.5 ug of each antibody. After incubation for 24 hours with IFNa (1pg/ul), PBMCs were assayed for IFNa-induced IFITM3 upregulation and CD14/CD16 levels and fractions by flow cytometry. For serum staining assays, PBMCs were cultured with media or 1-100 pg/ml IFNa for 38-46 hours. Samples were harvested and Fc receptors 
were blocked with unconjugated anti-CD16 (clone 3G8; BioLegend; \#302002), anti-CD32 (clone FUN-2; BioLegend; \#303202), and anti-CD64 (clone 10.1; BioLegend; \#305002) antibodies for $20 \mathrm{~min}$ on ice. Following one washing step with fluorescence-activated cell sorting (FACS) buffer (2\% fetal bovine serum, $1 \mathrm{mM}$ EDTA, PBS), non-specific binding of the detection antibody was blocked by incubating with unconjugated AffiniPure Donkey anti-human IgG (Jackson Immunoresearch; \#709-005-149) for $15 \mathrm{~min}$ at room temperature. After washing with FACS buffer, PBMCs were then stained for surface markers $30 \mathrm{~min}$ on ice. After staining incubation, cells were washed 3x times with FACS buffer (1500 rpm, 5 min, $4^{\circ} \mathrm{C}$ ) and incubated with $5 \mu$ autologous or clinical study participant sera for $30 \mathrm{~min}$ on ice. After washing the cells with FACS buffer, cell-bound antibodies were detected using an AffiniPure Donkey anti-human IgG-Alexa Fluor 647 antibody (Jackson Immunoresearch; \#709-605-149), which was incubated with the cells for 30 min on ice. Cells were washed again and resuspended in $1 \mu \mathrm{g} / \mathrm{ml}$ DAPI solution for live/dead discrimination. The following antibodies were used for flow cytometric analysis: anti-human CD3-BB700 (clone SK7; BD Biosciences; \#566575), anti-human CD14-BV711 (clone MSE2; BioLegend; \#301838), anti-human CD15-BV786 (clone W6D3; BD Biosciences; \#741013), anti-human CD16-BV605 (clone 3G8; BioLegend; \#302040), anti-human CD19-BV785 (clone HIB19; BioLegend; \#302240), anti-human CD45-APCeFluor780 (clone HI30; ThermoFisher; 470459-42), anti-human IFITM3-AlexaFluor 647 (clone EPR5242; Abcam; ab198573).

\section{PBMC Fc receptor crosslinking experiment:}

96 well flat bottom polystyrene plates were coated overnight at $4 \mathrm{C}$ with either 10 or $5 \mathrm{ug} / \mathrm{mL}$ of combinations of anti-CD16 (clone 3G8; BioLegend; \#302002), anti-CD32 (clone FUN-2; BioLegend; \#303202), and anti-CD64 (clone 10.1; BioLegend; \#305002) diluted in PBS. Plates were washed $3 \mathrm{x}$ with PBS prior to PBMC plating which were prepared as detailed above. 250k PBMC's per well were spun down briefly and incubated at 37C for 15 minutes to allow for coated antibody engagement. IFNa was then added into the well and cells incubated for 24 hours at $37 \mathrm{C}$ prior to flow cytometry as described above.

\section{Statistical Analysis and Data visualization:}

Statistical analyses were performed using GraphPad prism or the R software package. Null hypotheses between two groups were tested using the non-parametric Mann-Whitney test to account for non-normal distribution of the data. Likewise, for multiple groups, comparisons were made by two-way ANOVA or non-parametric Kruskal-Wallis test followed by multiple comparisons. The specific statistical tests and their resultant significance levels are also noted in each figure legend. The R packages Seurat, ggplot2 (version 3.1.0) (Wickham, 2016) GraphPad Prism and Adobe Illustrator were used to generate figures.

\section{Data and Code Availability Statement}

The data reported in this manuscript are in the main paper and in the supplementary materials. Cellranger-processed raw feature-barcode matrices are available at GEO using accession GSE163668 and raw fastq files for all 10X libraries are deposited in SRA. Scripts used to process all data along with relevant clinical information for each patient are available at https://github.com/UCSF-DSCOLAB/combes_et_al_COVID_2020. 


\section{Extended Data}
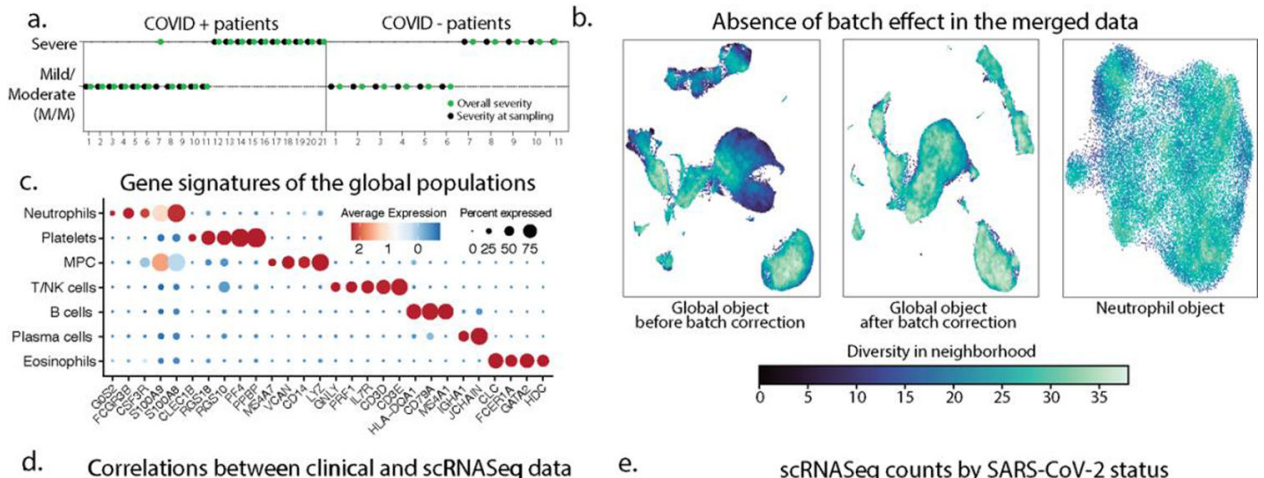

Correlations between clinical and scRNASeq data

e.

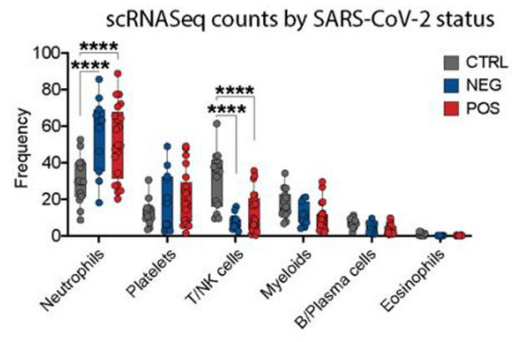

Extended Data 1: Immune phenotyping of patients admitted with respiratory symptoms using whole blood single-cell RNA sequencing.

a. Patient symptoms plot: symptom at day of sampling (first day of admission to the hospital) is represented in black, while symptom based on the entire course of hospitalization is in green. In the rest of the manuscript, we categorized patient into mild/moderate or severe cases based on all the entire course of hospitalization (green). b. Quantification of the batch effect using neighbor diversity score in the global object UMAP before (left) and after (middle) batch correction, along with the neutrophil (right) UMAP plot, as in Fig $1 \mathrm{~b}$ and Fig1c, using the diversity in neighborhood method. c. Dotplot representation of landmark genes expressed by global populations in Fig1b. d. Spearman's correlation comparison between disease severity and population frequencies calculated from 10X scRNAseq analyses (10X) or complete blood cell counts (CBC). Patients for which $\mathrm{CBC}$ counts were unavailable were excluded. Significance was calculated using Spearman's method. * p value $<0.05 ; * *$ p value $<0.05 ; * * * \mathrm{p}$ value $<0.005(\mathrm{n}=29)$ e. Frequency of the global populations in Fig1b among all cells across SARS-CoV-2 status (control, n=14; NEG, $\mathrm{n}=11 ; \mathrm{POS}, \mathrm{n}=21$ ). 

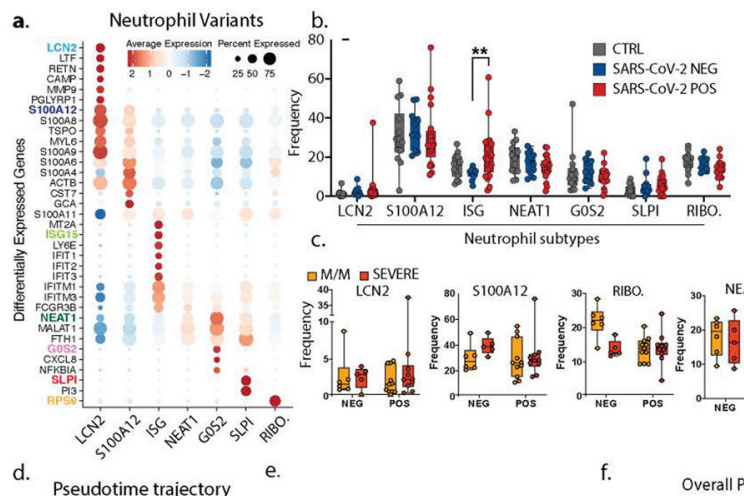

c.

Neutrophil subtypes $\square M / M$
口 SEVVERE
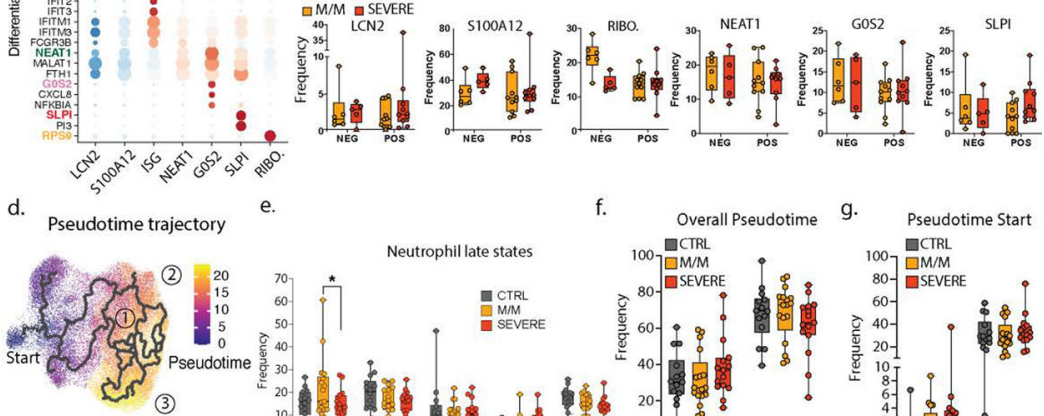

f. Overall Pseudotime g.

Neutrophil late states
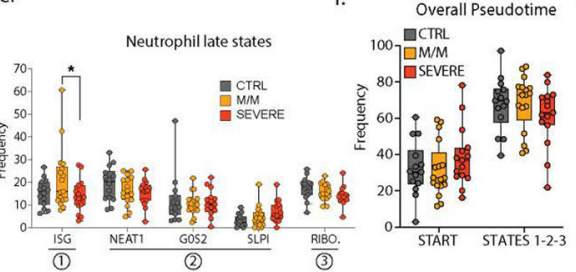

Pseudotime Start
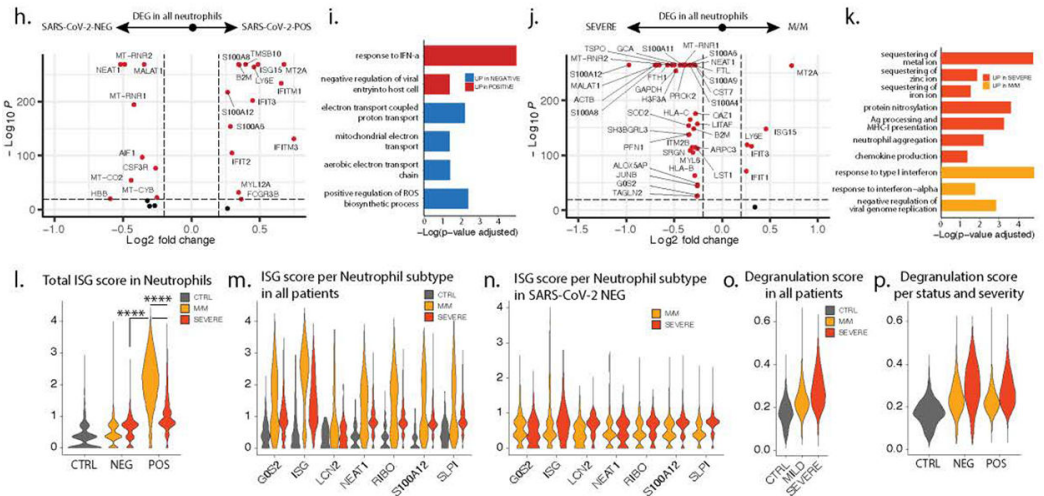

Extended Data 2: Patients with severe COVID-19 lack IFN response in neutrophils.

a. Dotplot representation of top differentially-expressed-genes (DEG) between neutrophil subsets. b. Frequencies of neutrophil subsets among all neutrophils across control $(n=14)$, SARS-CoV-2 negative $(n=11)$ and SARS-CoV-2 positive $(n=21)$ individuals. c. Frequency of the LCN2, S100A12, RIBO., NEAT1, G0S2 and SLPI neutrophils among all neutrophils across SARS-CoV-2 status and disease severity (NEG M/M, n=6; NEG severe, $n=5$; POS M/M, n=11, POS severe $n=10$ ). d. Pseudotime trajectory of neutrophil subsets. e. Frequencies of the neutrophil subsets among all neutrophils at later stages of pseudotime trajectories across control $(n=14)$, mild/moderate $(n=17)$ and severe $(n=15)$ individuals. $f$ and g. Frequencies of the neutrophil subsets among all neutrophils across control $(n=14)$, mild/moderate $(M / M, n=17)$ and severe $(n=15)$ individuals at the overall start/late states of the trajectories (f) or at specific early stages of the pseudotime (g). h to k. Volcano plots showing DEG ( $h$ and $j$ ) and bar plots showing GO term enrichment from these DEG (i and k) between all neutrophils from either SARS-CoV-2 positive vs negative patients ( $h$ and i) or mild/moderate vs severe patients ( $\mathrm{j}$ and $\mathrm{k}$ ). $\mathbf{I}$ to $\mathbf{p}$. Scores of ISG signature (l to $\mathrm{n}$ ) and neutrophil degranulation (o and $\mathrm{p}$ ) in either all neutrophils across control, mild/moderate an severe patients (I and o), all neutrophils across SARS-CoV-2 status and disease severity $(\mathrm{m}$ and $\mathrm{p}$ ) or specific neutrophil subtypes across severity in either all patients $(\mathrm{m})$ or only 
SARS-CoV-2 negative patients (n). Statistical significance was assessed using a two-way ANOVA test with multiple comparisons for panels c, e and g, and using a two-tailed Wilcoxon test for panel 1. * p.value $<0.05$; ** p.value $<0.01$; *** p.value $<0.001$; **** p.value $<0.0001$. Boxplot center, median; box limits, $25^{\text {th }}$ and $75^{\text {th }}$ percentile; whiskers, min. and max. data point.

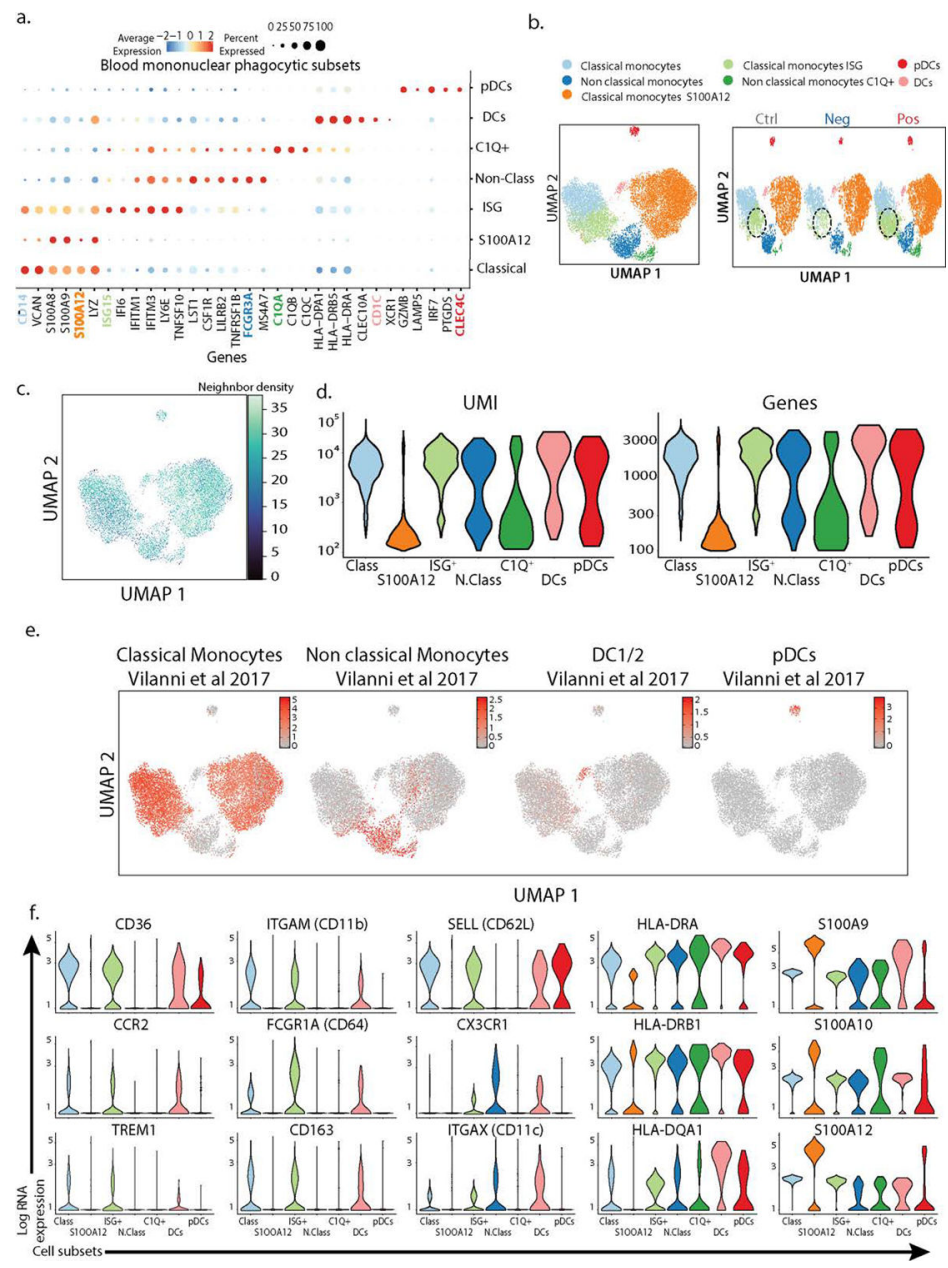

Extended Data 3. Characterization of peripheral blood mononuclear phagocytes subsets in our cohort.

a. Dotplot representation of the top differentially-expressed-genes (DEG) between clusters identified in blood mononuclear phagocytic cell (MPC) subsets. b. UMAP visualization of the 19,289 MPC isolated from the entire dataset (left) and split by SARS-CoV-2 status (right). c. Quantification of the batch effect before and after batch correction using neighbor diversity score in the mononuclear phagocytic cells (MPC) object from UMAP plot in (b), using the diversity in neighborhood method. d. Violin plot of number of unique genes (bottom) and number of unique molecules (top) detected from Single cell sequencing for each cluster identified in the MPC dataset. e. Overlay of previously described blood mononuclear phagocytic cell signature from healthy individual (38) on MPC from UMAP plot in (b). f. Violin plots of canonical genes previously described as expressed by blood MPC for each for each cluster identified in the MPC dataset. 
a.

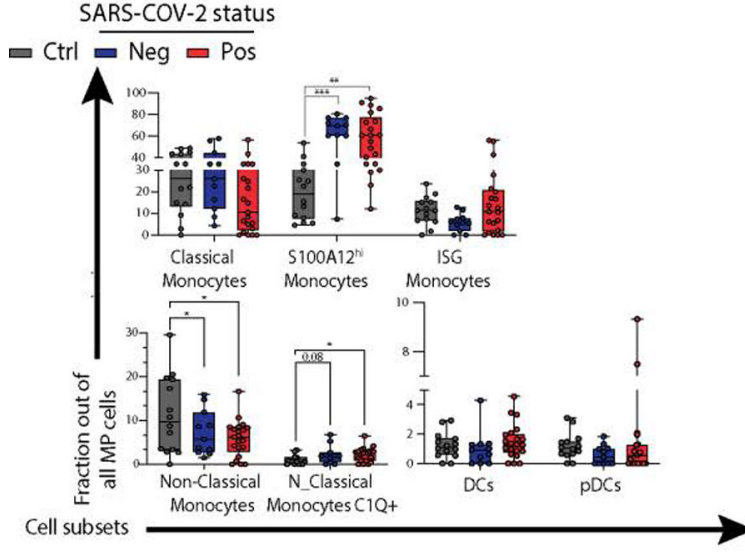

d.
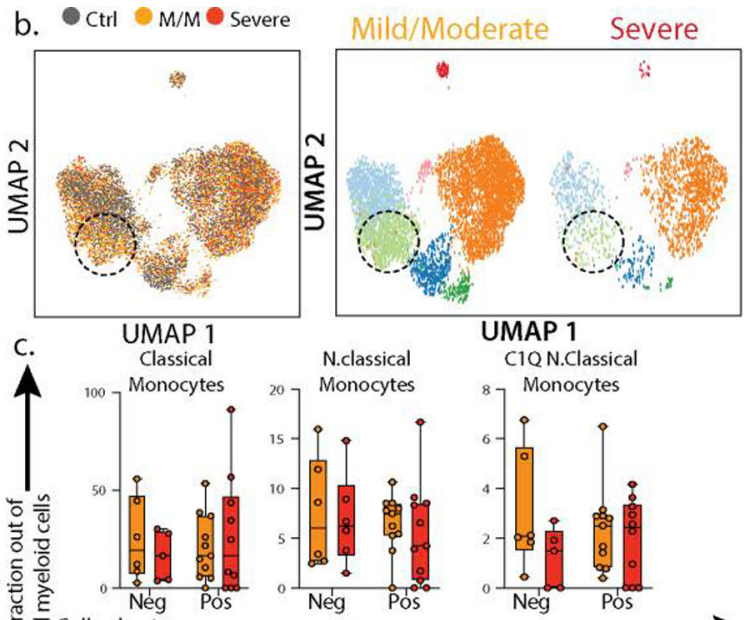

UMAP 1

C1Q N.Classical

$\left.{ }_{8}\right]$ Monocytes
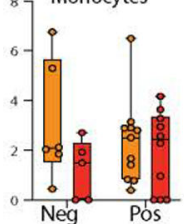

lassical monocytes Classical monocytes ISG pDCs

- Non classical monocytes

Classical monocytes S100A12

Non classical monocytes $\mathrm{C1Q}+\mathrm{DCs}$

Glycolysis

OXPHOS

Argüello et al 2020

Argüello et al 2020

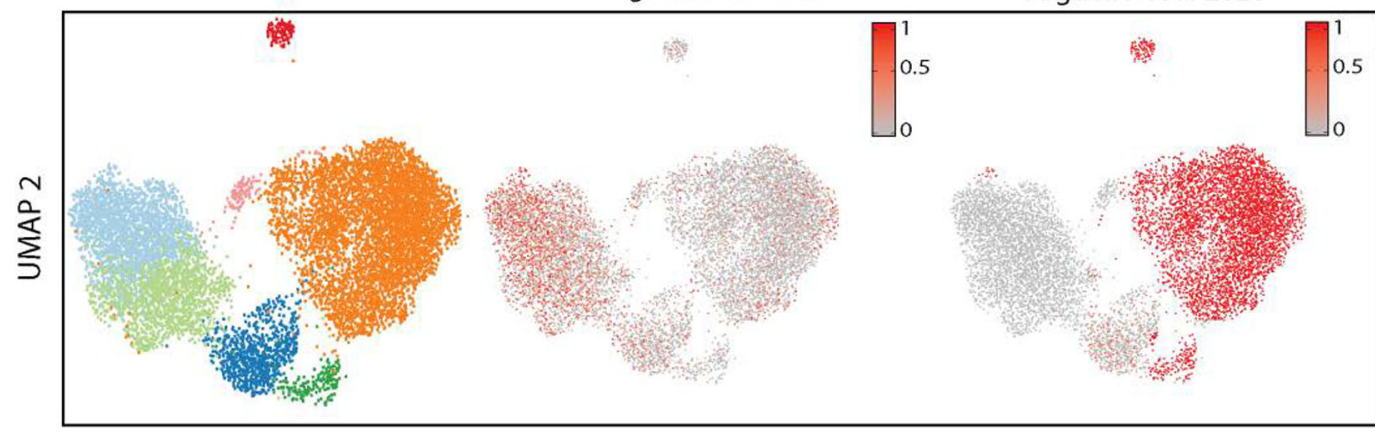

e.

Differential gene expression on all monocluear phagocytic cells

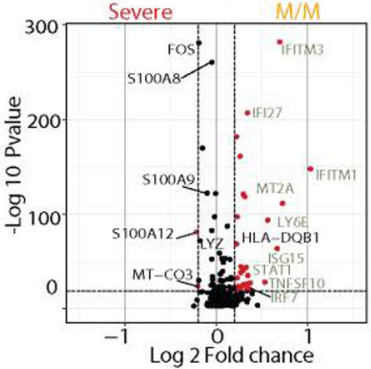

f.

Mono-DCs ${ }^{-1}-0.500 .5$

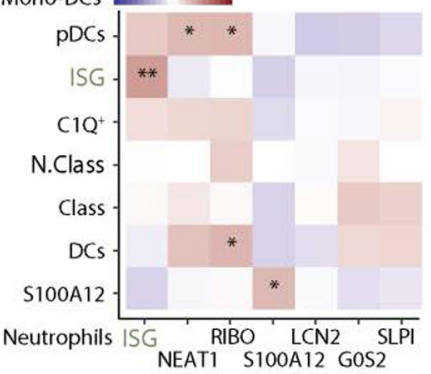

g.

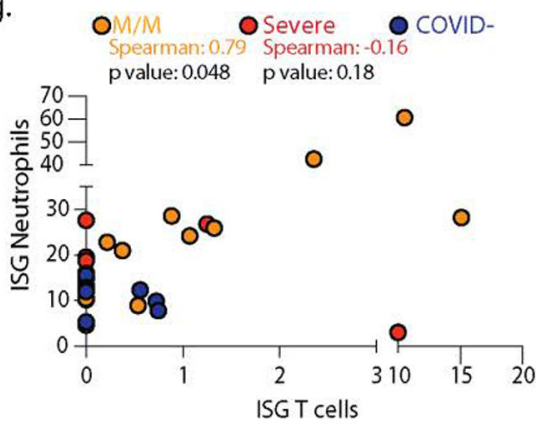

Extended Data 4: Severe COVID-19 is defined by the lack of a concerted IFN response across multiple cell types.

a. Frequencies of the MPC subsets among all MPC across control $(n=14)$, SARS-CoV-2 negative $(n=11)$ and SARS-CoV-2 positive $(n=21)$ individuals. b. UMAP visualization of the 19,289 MPC colored (left) and split by (right) by disease severity. c. Frequencies of the classical monocytes, cycling monocytes, non-classical monocytes and $\mathrm{C} 1 \mathrm{Q}+$ non classical monocytes among all MPC across SARS-CoV-2 negative (M/M, n=6; severe, $\mathrm{n}=5)$ and SARS-CoV-2 positive (M/M, $\mathrm{n}=11$; severe, $\mathrm{n}=10)$ individuals split it by disease severity. d. Overlay of previously described (39) glycolytic and oxidative phosphorylation 
gene signature on mononuclear phagocytic cells (MPC) from UMAP plot in FigS3b. e. Volcano plot showing results of differential gene expression (DGE) analysis performed on all MPC between mild/moderate (right) and severe (left) patients. f. Correlation matrix using Spearman Rank Correlation between the frequency of all neutrophils and monocytes subtypes in all SARS-CoV-2 negative $(\mathrm{n}=11)$ and SARS-CoV-2 positive patients $(\mathrm{n}=21)$. g. Scatter plot between neutrophil and CD4 T cell ISG positive subsets patient by patient $(\mathrm{M} / \mathrm{M}, \mathrm{n}=11$; severe, $\mathrm{n}=10$; COVID-, $\mathrm{n}=11$ ). Statistical significance was assessed using a two-way ANOVA test with multiple comparisons. * p.value $<0.05$; ** p.value $<0.01$; *** p.value $<0.001$; **** p.value $<0.0001$. Boxplot center, median; box limits, 25 th and 75 th percentile; whiskers, min. and max. data point.
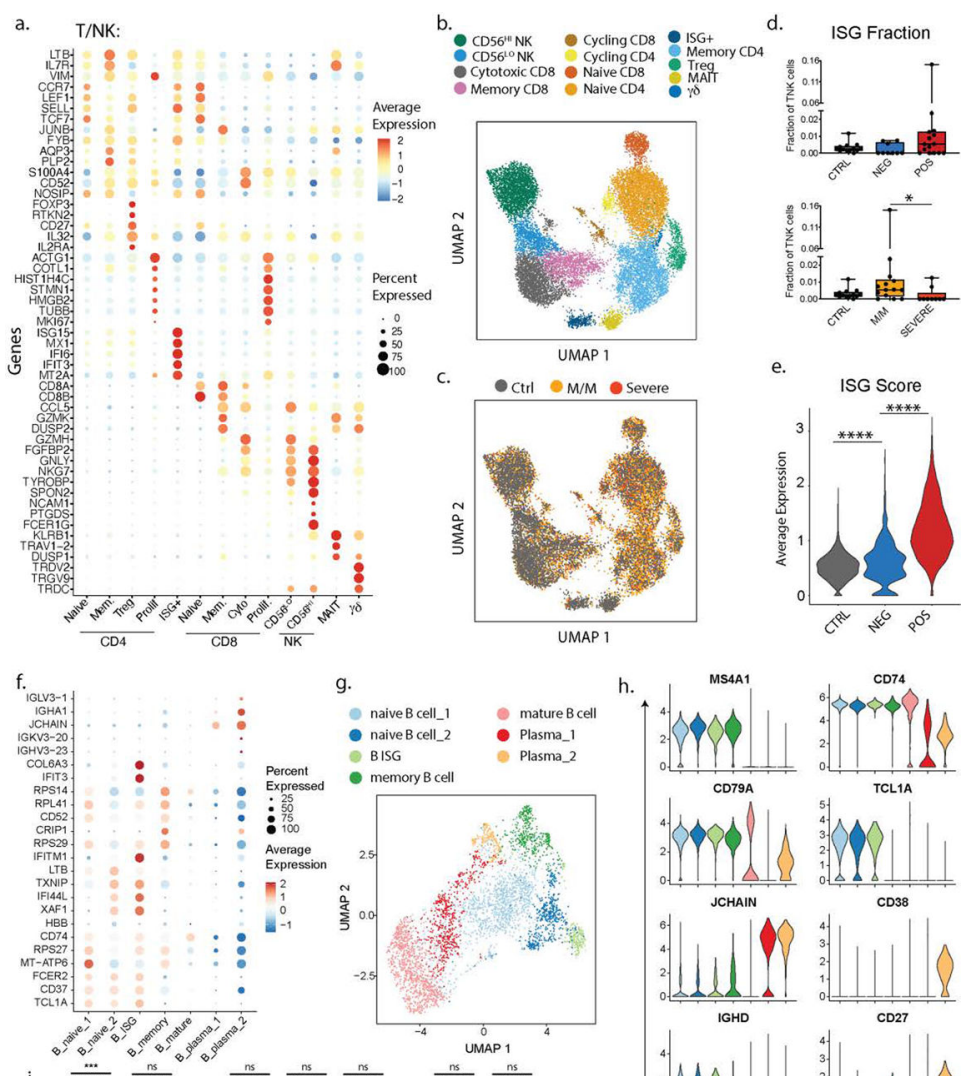

g.
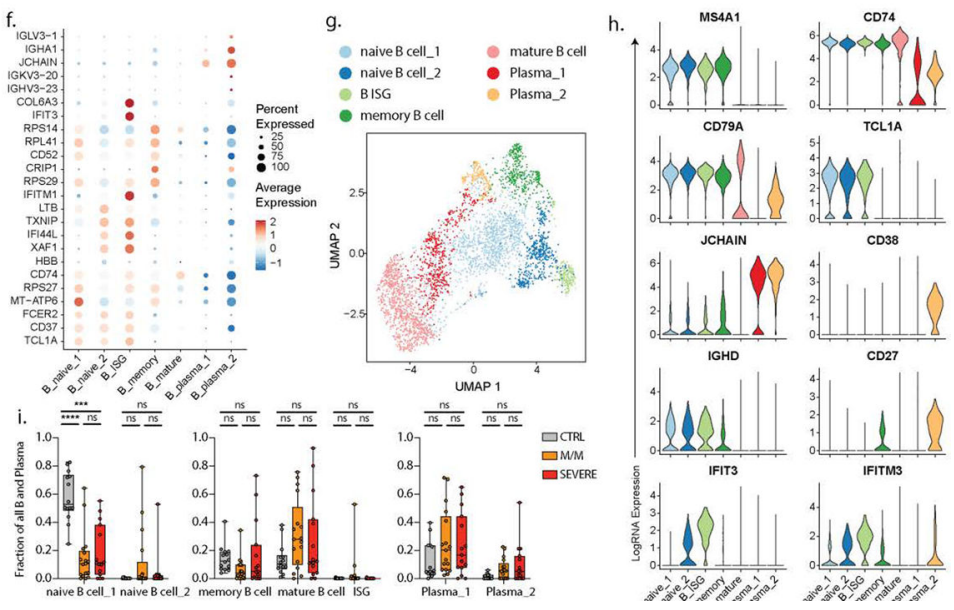

Extended Data 5: Characterization of the peripheral blood $T$ and $B$ lymphocytes subsets in our cohort.

a. Dotplot representation of the top DEG between clusters identified in the T and NK cell subset. b. UMAP visualization of 16,708 $\mathrm{T}$ and NK cells in the entire dataset showing various subsets, colored distinctly by their identity. c. Overlay of the above UMAP of all $\mathrm{T}$ and NK cells, colored by disease severity underlining the lack of batch effects while merging the datasets from all patients. d. Abundance of the Interferon-stimulated-gene (ISG)+ subset among all $\mathrm{T}$ and NK cells in healthy donors ( $\mathrm{n}=13$ ), SARS-CoV-2 negative 
$(\mathrm{n}=9)$ and SARS-CoV-2 positive $(\mathrm{n}=15)$ patients (top) and in healthy donors and patients with mild/moderate $(\mathrm{M} / \mathrm{M}, \mathrm{n}=14$ ) and severe disease (bottom, $\mathrm{n}=9$ ). e. ISG signature score between healthy controls, SARS-CoV-2 negative and SARS-CoV-2 positive patients. f. Dotplot representation of the top DEG between clusters identified in the B and plasma cell subset. g. UMAP visualization of 4,380 B and plasma cells isolated from the entire dataset showing various subsets, colored distinctly by their identity. h. Violin plots of canonical genes previously described as expressed by B and plasma cells for each identified cluster. i. Frequencies of the identified clusters among all B and plasma cells in healthy donors $(n=14)$ and patients with $M / M(n=17)$ and severe disease $(n=15)$. Differences in d. and e. were calculated using Kruskal-Wallis test. $* \mathrm{p}<0.05$ and $* * * * \mathrm{p}<0.001$. Differences in i. were calculated using a two-way ANOVA test with multiple comparisons. * p.value $<0.05$ and $* * * *$ p.value $<0.0001$. ns, non-significant. Boxplot center, median; box limits, $25^{\text {th }}$ and $75^{\text {th }}$ percentile; whiskers, min. and max. data point.

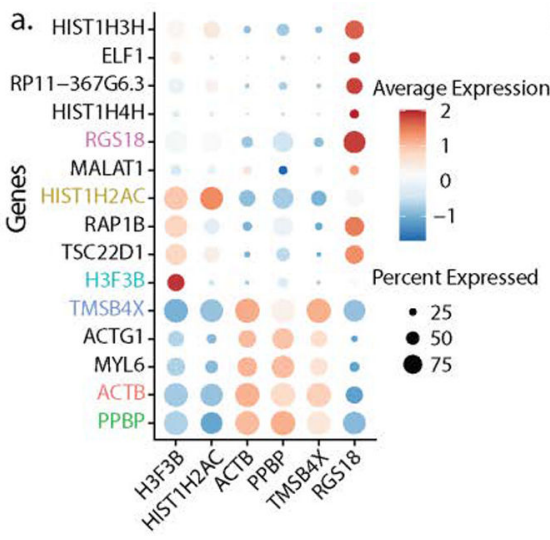

d.

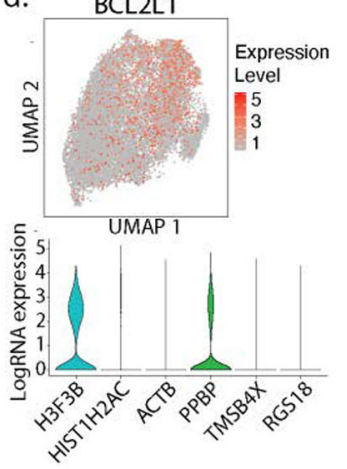

e.

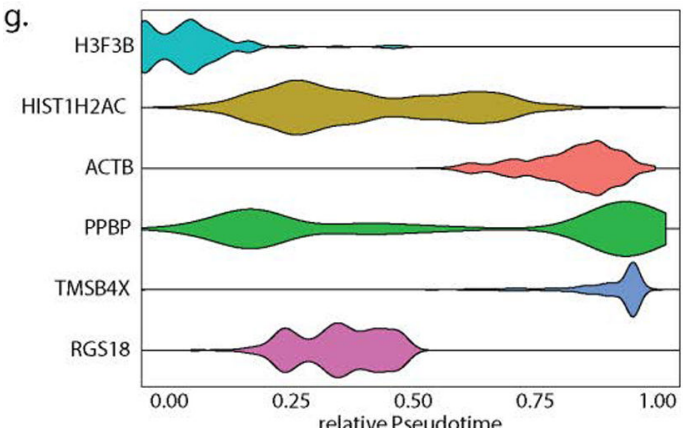

HIST1H2AC platelet

- ACTB platelets

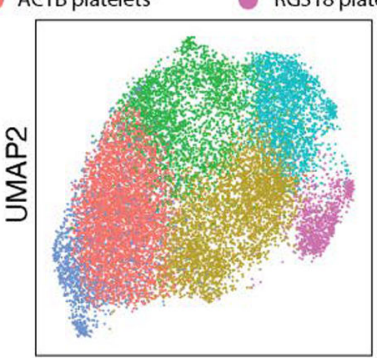

UMAP1
PPBP platelets

TMSB4X platelets RGS18 platelets

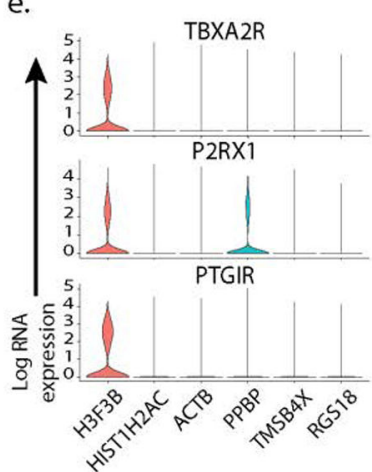

h.

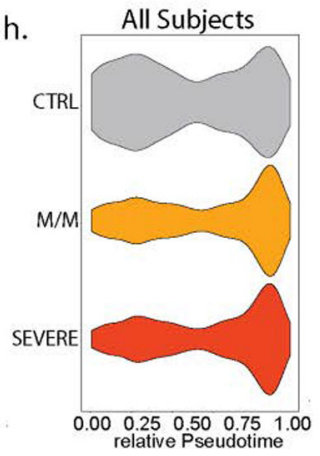

f.
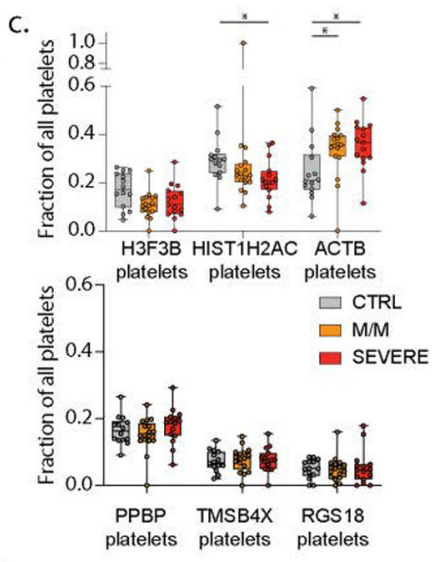

f. All Subjects

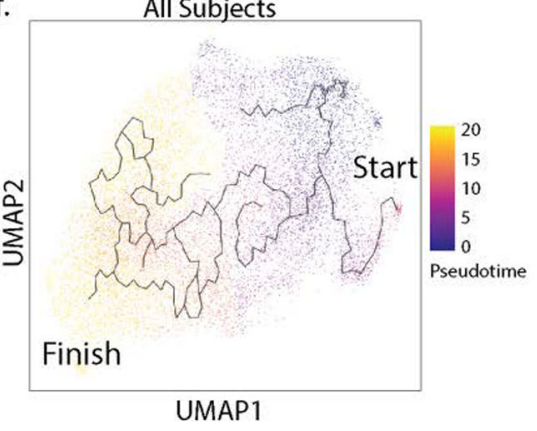

i.

ISG score

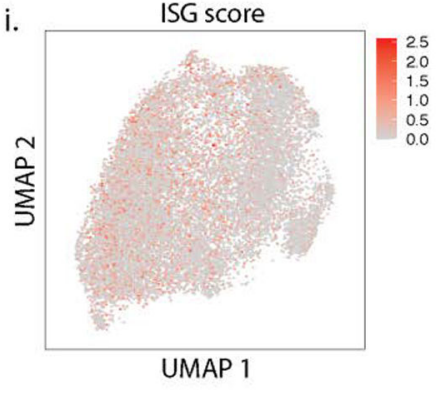

Nature. Author manuscript; available in PMC 2021 November 04. 
Extended Data 6: Characterization of the peripheral blood platelets subsets in our cohort. a. Dotplot representation of the top DEG between clusters identified in the platelet subset. b. UMAP visualization of 16,903 platelets isolated from the entire dataset showing various subsets, colored distinctly by their identity. c. Frequencies of the identified clusters among all platelets in controls $(n=14)$ and all patients with mild/moderate $(M / M, n=17)$ and severe disease $(\mathrm{n}=15)$. d. UMAP visualization of all platelets colored by BCL2L1 (top) and violin plot of $B C L 2 L 1$ expression level across all identified platelet subsets. e. Violin plots of genes identifying young, reticulated platelets (9) in the platelet dataset. f. UMAP visualization of all platelets with overlay of Pseudotime trajectory. g. Violin plots of the relative pseudotime of each platelet cell subset present in Figure $3 \mathbf{b} \mathbf{~ h}$. Violin plot of the relative Pseudotime of all platelets split by healthy donors, mild/moderate and severe patients. i. UMAP visualization of all platelets colored by ISG score. Differences in c. were calculated using a two-way ANOVA test with multiple comparisons. *p.value <0.05; $* *$ p.value $<0.01 ; * * *$ p.value $<0.001 ; * * * *$ p.value $<0.0001$; ns: non-significant. Boxplot center, median; box limits, $25^{\text {th }}$ and $75^{\text {th }}$ percentile; whiskers, min. and max. data point.
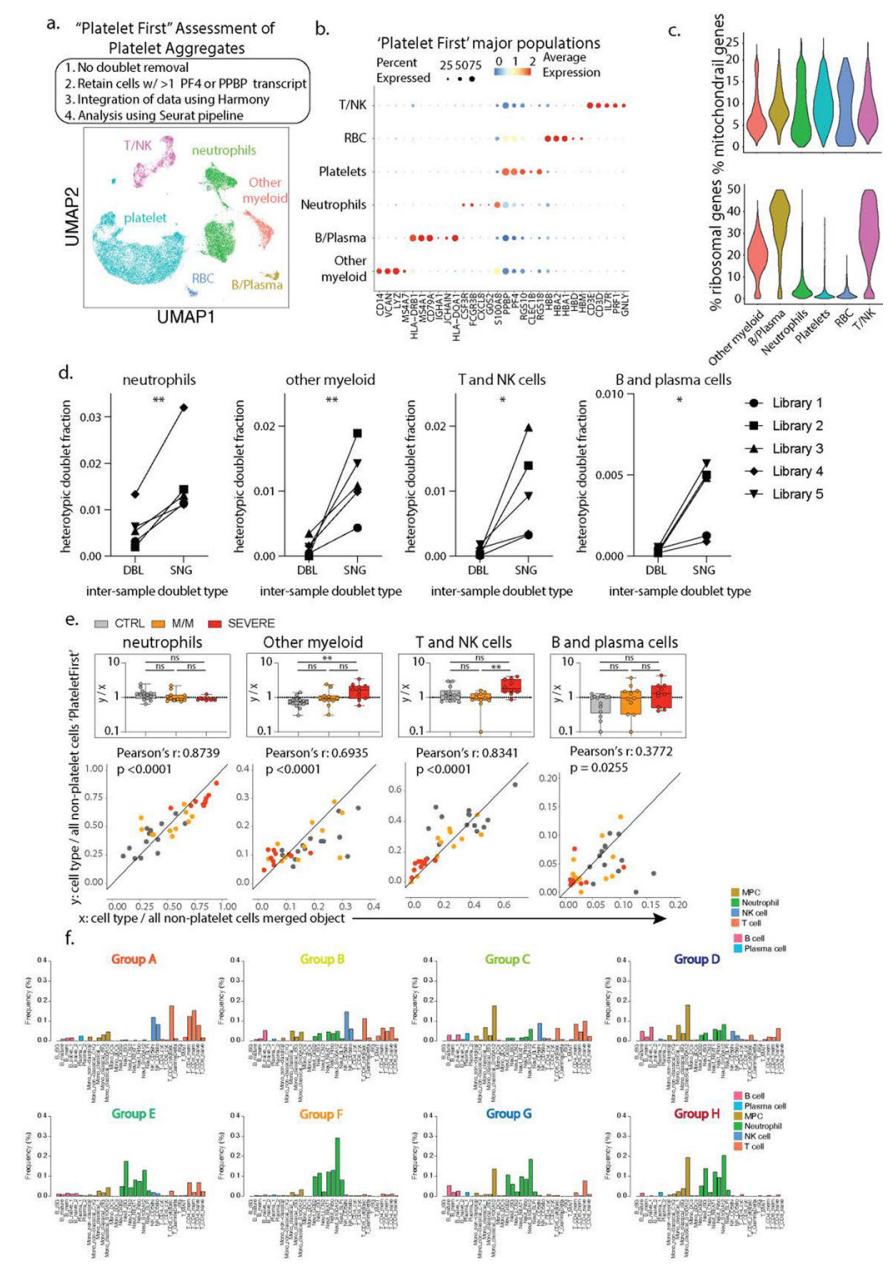

Extended Data 7: Leveraging single-cell RNA sequencing to assess platelets aggregates and define immune states in COVID-19 patients. 
a. Outline of 'Platelet First' assessment to identify platelet aggregates in entire whole blood scRNA-seq data set. UMAP visualization of the 52,757 putative platelet aggregates with specific populations overlaid. b. Dotplot representation of the top DEG between clusters identified in the 'Platelet First' object. In this object no doublet removal filtering step was applied to include all heterotypic cell-cell aggregates (Step 1). This was followed by retaining all cells with $>1$ platelet-specific transcripts PF4 or PPBP (Step 2). Step 2 guaranteed analysis of cell events and aggregates containing platelets. Identically to our original data set in Figure 1b, integration of data was done using Harmony (Step 3), and the 'Platelet First' object was then analyzed using the Seurat v3 pipeline (Step 4). c. Violin plots of the percentage of mitochondrial and ribosomal genes within clusters identified in the 'Platelet First' object. d. Inter-sample doublet rates in inferred platelet-involved heterotypic doublets show that platelet aggregates occur in vivo. DBL, doublet, $\mathrm{n}=5$ libraries. SNG, singlet, $\mathrm{n}=5$ libraries. e. Bottom: Scatter plot of cell type frequency within merged object of entire cohort shown in Figure 1b ( $x$-axis) versus same cell type frequency within 'Platelet First' object ( $y$-axis). The identity line $x=y$ is drawn as a reference. Each dot represents a control $(\mathrm{n}=14)$ or SARS-CoV-2 positive patient sample and are color-coded by disease severity $(M / M, n=11$; severe, $n=10)$. Pearson $r$ correlation coefficient and two-tailed $p$ value are shown for each cell type. Top: Box plots of $y / x$-ratio for each healthy control or patient sample, separated by disease severity. f. Cell fraction histograms representing bin-wise mean of relative frequency (i.e., cell fraction) of each immune cell subtype for all patients in a given group, colored as described in Fig2f. Differences in d. were calculated using a one-sided Student's t test * p.value $<0.05$ and $* *$ p.value $<0.01$. Differences in e. were calculated using a two-way ANOVA test with multiple comparisons. *p.value $<0.05$; $* *$ p.value $<0.01 ; * * *$ p.value $<0.001 ; * * * *$ p.value $<0.0001$; ns: non-significant. Boxplot center, median; box limits, $25^{\text {th }}$ and $75^{\text {th }}$ percentile; whiskers, min. and max. data point. 


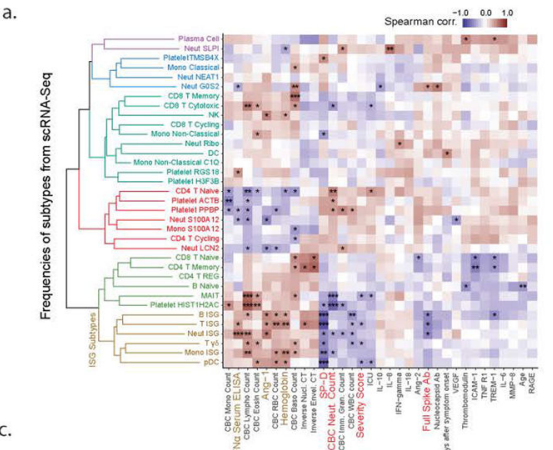

b.

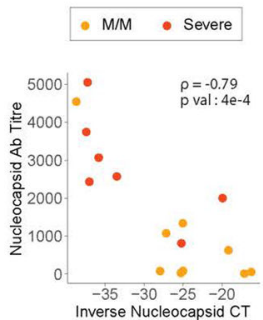

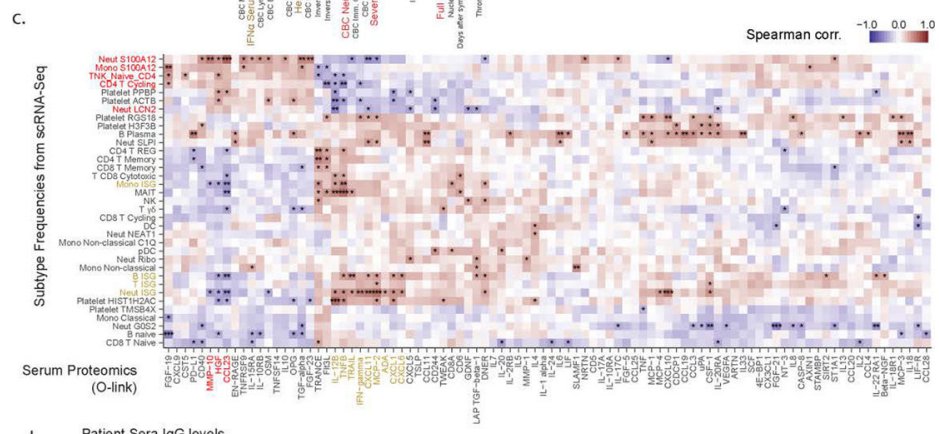
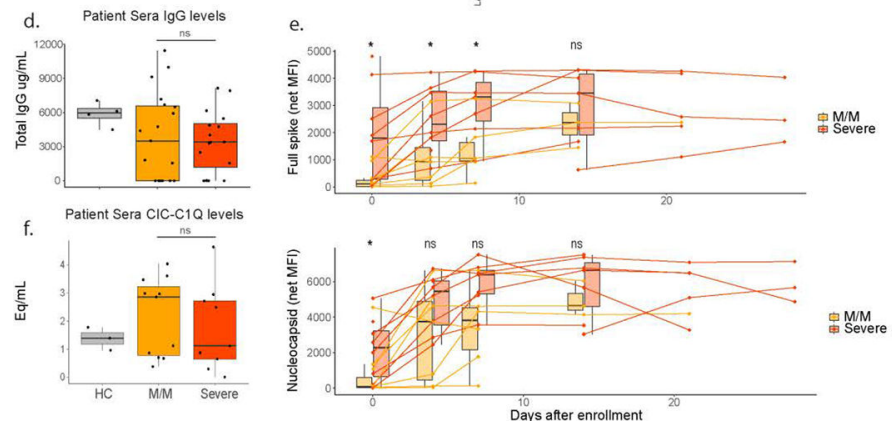

Extended Data 8: Holistic assessment of COVID-19 peripheral blood profile combining singlecell RNA sequencing, clinical blood counts and cytokines plasma levels.

a. Matrix of Spearman correlation coefficients between all subtype frequencies (out of major cell types, e.g. Neut ISG out of all Neutrophils) obtained from scRNA-Seq versus patient metadata, viral load, Ab titers, and serum analyte levels on a patient-by-patient basis excluding healthy controls. Patients for which data were unavailable were excluded from correlation analysis for each comparison. Variables on both axes were ordered via hierarchical clustering with the computed dendrogram displayed for subtype frequencies. This dendrogram was divided into 6 groupings with the one containing ISG+ subtypes highlighted in brown. Clinical variables generally correlated with severity highlighted in red and anti-correlated in brown. ( $\mathrm{n}$ for correlation comparisons ranged from $\mathrm{n}=14-32$ individuals) $* \mathrm{p}<0.05, * * \mathrm{p}<0.005$, $* * * \mathrm{p}<0.0005$. b. Scatter plots showing viral load versus levels of antibody binding SARS-CoV-2 Nucleocapsid protein for patients in the cohort with severity overlaid. Antibody levels are shown as arbitrary units of MFI from Luminex assay while viral load is represented by an inverse CT number from QRT-PCR with target amplification of the SARS-CoV2 Nucleocapsid sequence. Correlation coefficient and significance calculated using Spearman's method. Patients for which data was unavailable were excluded (M/M, n=9; severe, $n=7$ patients). c. Matrix of Spearman correlation coefficients between all subtype frequencies (out of major celltypes e.g. Neut ISG represents 
$\%$ out of all Neutrophils) obtained from scRNA-Seq versus protein analyte abundance in plasma as measured using Olink assay on a patient-by-patient basis excluding healthy controls. Patients for which data were unavailable were excluded from correlation analysis for each comparison. Variables on both axes were ordered via hierarchical clustering. ISG subtypes and protein levels strongly correlated with their frequency highlighted in brown. Subtypes and proteins strongly anti-correlated with ISG+ subtypes highlighted in red. ( $\mathrm{n}=31$ for all comparisons). $* \mathrm{p}<0.05, * * \mathrm{p}<0.005, * * * \mathrm{p}<0.0005$. d. Computed total $\operatorname{IgG}$ levels in patient sera from ELISA absorbance readings. ( $n=4 / 19 / 16$ for $\mathrm{HC} / \mathrm{MM} / \mathrm{Severe}$ ) e. Longitudinal measurements of anti-Spike and Nucleocapsid antibody levels in patient sera at the indicated days post-enrollment in study. Connected points represent tracking of a single individual. ( $\mathrm{n}=11 / 8 / 7 / 8 / 6 / 7 / 3 / 7 / 1 / 5 / 0 / 3$ for MM vs. Severe for D0,4,7,14,21,27 respectively) f. Levels of circulating immune complexes (CIC) in patient sera as measured by ELISA with human C1Q used to capture CIC's and an anti-hIgG secondary. Levels shown as heat aggregated human gamma globulin equivalents per $\mathrm{mL}$ or $(\mathrm{Eq} / \mathrm{mL})$. $(\mathrm{n}=3 / 11 / 9$ for HC/MM/ Severe respectively). Boxplots represent 25/50/75 percentiles. Statistical testing performed using two-sided Wilcoxon rank-sum test. Boxplot center, median; box limits, $25^{\text {th }}$ and $75^{\text {th }}$ percentile; whiskers, min. and max. data point. 
a.

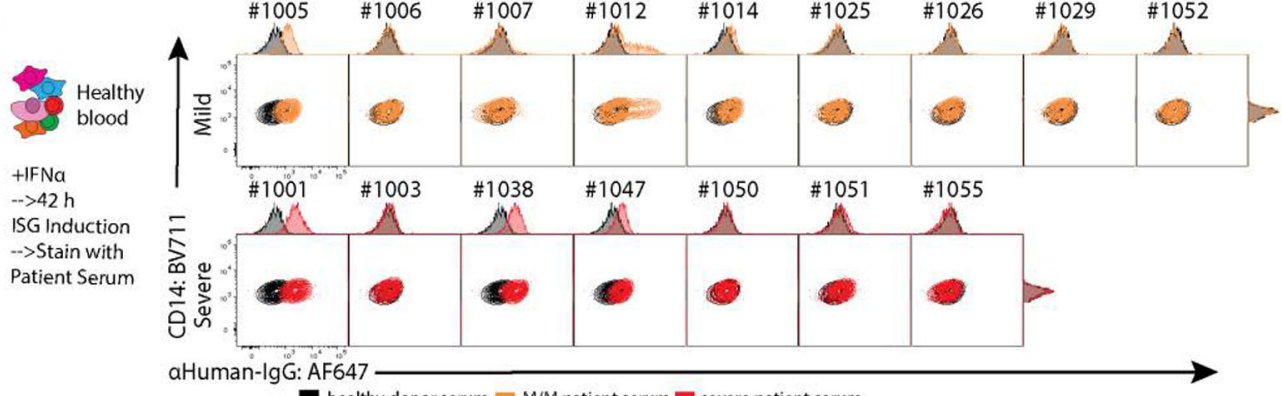

b.

C.

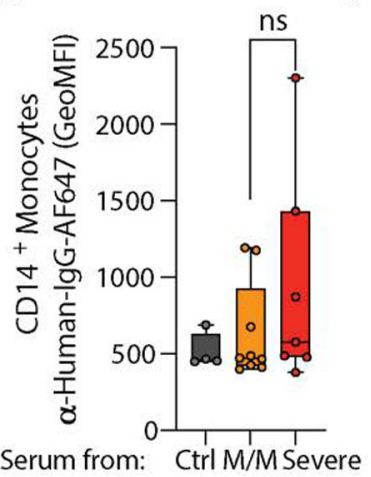$$
\begin{array}{|l|}
\hline \\
\hline \text { Coner } \\
\hline 1005 \\
1006 \\
1007 \\
1012 \\
1014 \\
1025 \\
1026 \\
1029 \\
1052 \\
\hline 100 \\
1003 \\
10 \\
10 \\
1050 \\
1051 \\
1055 \\
\hline
\end{array}
$$

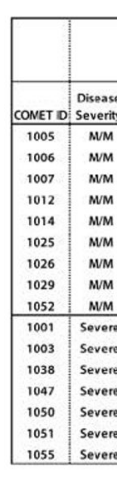

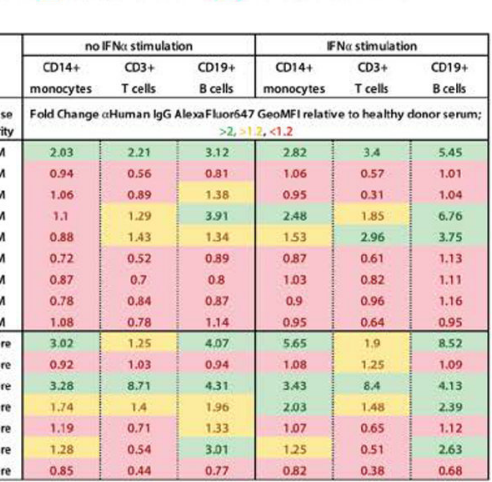

d.

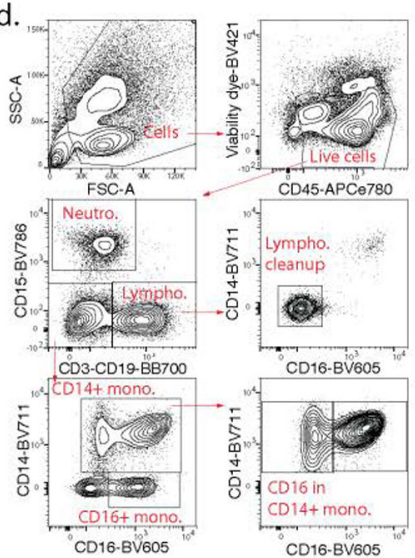

e.

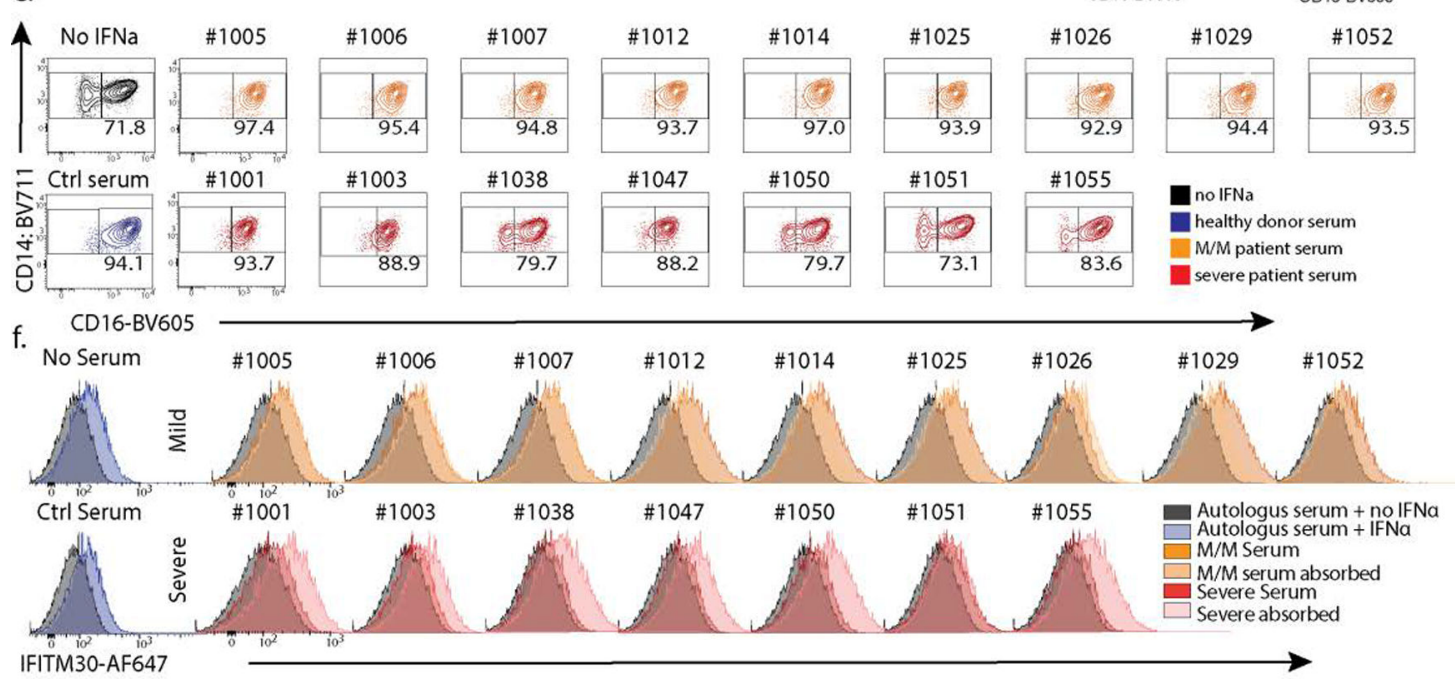

Extended Data 9: Staining and Neutralization assay on IFN-stimulated heathy PBMC using COVID-19 patients' serum.

a. Contour plots and histograms of CD14+ monocytes from healthy donor blood cultured with IFNa to induce expression of ISGs and stained with serum from heathy donor, mild/moderate $(\mathrm{M} / \mathrm{M})$ or severe SARS-CoV-2 positive patients with secondary staining with anti-human IgG. b. Geometric MFI of serum staining on CD14+ monocytes treated with IFNa, quantifying data in Figure S7A. Ctrl, n=4; M/M, n=9; severe, $n=7$. Boxplot center, median; box limits, 25th and 75th percentile; whiskers, min. and max. data point. c. Summary table of serum staining experiment. Fold change (FC) of anti-Human IgG AlexaFluor647 GeoMFI relative to allogeneic healthy donor serum on non-stimulated and IFNa-stimulated healthy PBMCs is listed for each analyzed cell type. Table data cells are 
color-coded based on degree of FC; green, $\mathrm{FC}>2$; yellow, $2>\mathrm{FC}>1.2$; red, $\mathrm{FC}<1.2$., d. Gating strategy for PBMCs to identify different subpopulations. e. Modulation of intermediate to classical CD14 monocytes transition by mild/moderate (orange) and severe (red) patient serum. Each plot represents a single serum sample. Representative experiment from three independent trials and two different healthy PBMC donors. f. Histograms of IFITM3 expression by CD3+ CD19+ lymphocytes from healthy donor cultured with IFNa and serum from heathy donor (blue), mild/moderate (orange) and severe (red) SARS-CoV-2 positive patients. Mild/Moderate (light yellow) or Severe (pink) sera were pre-treated with protein G/A before incubation with PBMC. Each plot represents a single serum sample. Representative experiment from two independent trials and two different healthy PBMC donors. For a, b, c, e, f, data from one of two representative experiments is shown. ns, non-significant. 

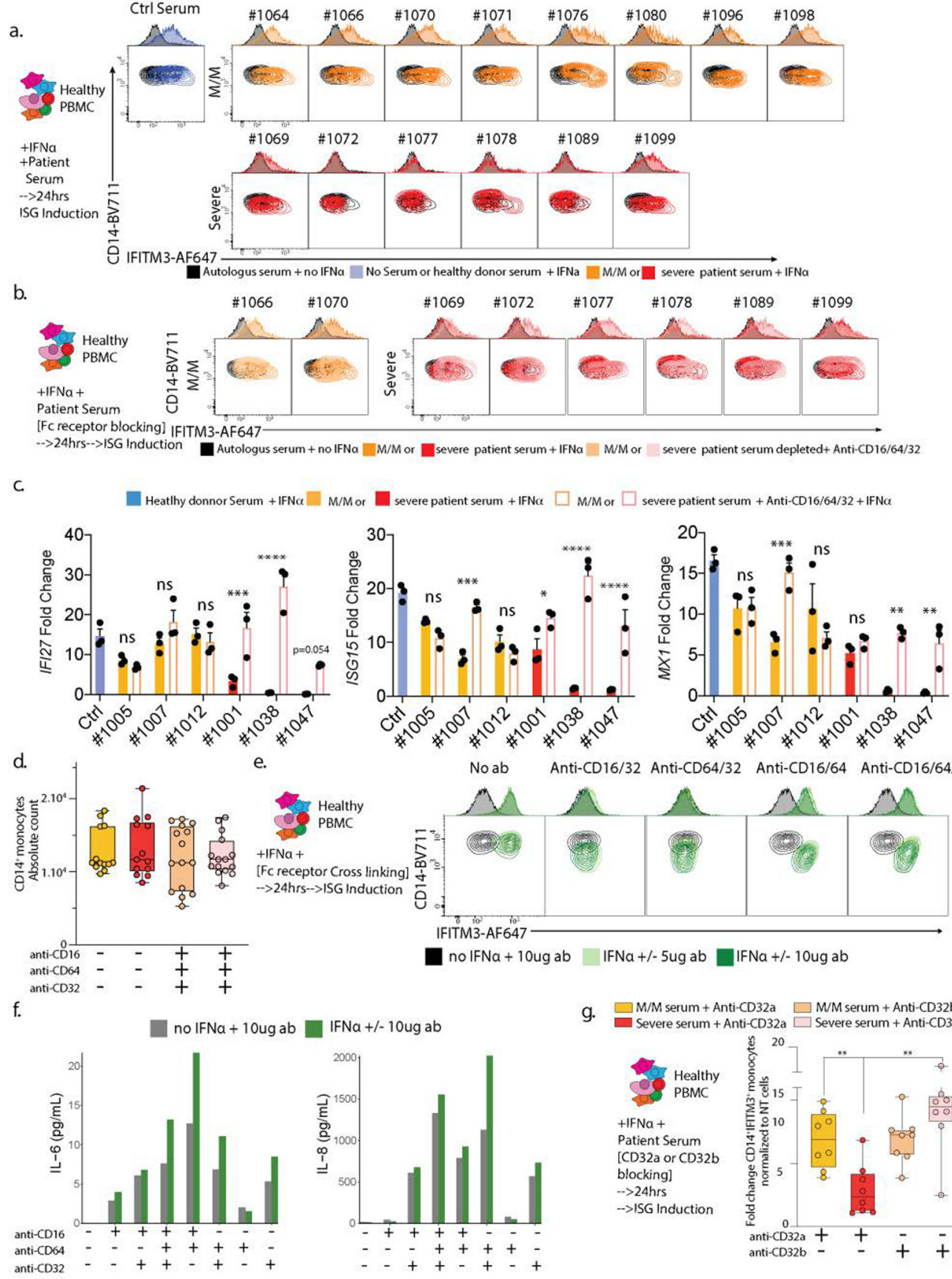

Noab Anti-CD16/32 Anti-CD64/32 Anti-CD16/64 Anti-CD16/64/32

Extended Data 10: Antibodies present in severe COVID-19 patients antagonize IFNAR Signaling through FC $\gamma$ RIIb.

a. Test of ISG neutralization by M/M or severe serum as presented in Figure 3e, here using sera from a validation cohort of patients. b. Test of ISG neutralization by $\mathrm{M} / \mathrm{M}$ or severe serum in presence of anti-CD16/CD32/CD64 antibodies to block Fc receptors as presented in Figure 4a, here using sera from a validation cohort of patients. c. qPCR analysis of IFI27, ISG15 and $M X 1$ gene expression in healthy donor PBMCs treated with IFNa with the addition of $\mathrm{M} / \mathrm{M}$ or severe patient sera with or without Fc receptor blocking (Figure 4a). Fold changes are relative to untreated healthy donor PBMCs. $n=3$ /group. Data is plotted as 
mean \pm SEM. d. Absolute counts of CD14+ monocytes from experiments presented in Figure 4a ( $n=16$ /group). e and f. Contour plots and histograms of CD14 and IFITM3 expression by monocytes (e) and quantification by Luminex of IL-6, IL-8 in the supernatant (f) from the experiment presented in Figure $4 \mathrm{~b}$ and c. g. Boxplots showing fold changes of percentage of IFITM3 positive CD14+ monocytes upon IFNa stimulation normalized to non-treated cells ( 1 experiment on 2 different pbmc donors: $n=8$ /group). Differences in $\mathrm{c}$ and $\mathrm{g}$ were calculated using a two-way ANOVA corrected for multiple comparison. * p.value < 0.05 ; $* *$ p.value $<0.01$; *** p.value $<0.001$; **** p.value $<0.0001$; ns non-significant. Boxplot center, median; box limits, $25^{\text {th }}$ and $75^{\text {th }}$ percentile; whiskers, min. and max. data point.

\section{Supplementary Material}

Refer to Web version on PubMed Central for supplementary material.

\section{Acknowledgements}

We thank all members of the Krummel Lab and ImmunoX for discussion and guidance while developing this study. We would like to thank Dr Gaia Andreoletti for discussion and guidance on computational analysis. This work was supported by funds from the UCSF ImmunoX Initiative and funds from the NIH (R01 AI52116-S1 (MFK) 3U19AI077439-13S1 (DJE), NHLBI R35 HL140026(CC)). K.H.H. is supported by the American Cancer Society Postdoctoral Fellowship (\#133078-PF-19-222-01-LIB). A.R. is a Cancer Research Institute Irvington Postdoctoral Fellow supported by the Cancer Research Institute (Award \# CRI2940). This project has been made possible in part by grant number 2019-202665 from the Chan Zuckerberg Foundation.

\section{COMET Consortium group}

\begin{tabular}{|c|c|}
\hline Name & Institution \\
\hline Cathy Cai & ${ }^{1}$ Department of Pathology and ${ }^{2}$ ImmunoX, UCSF, San Francisco, California, USA. \\
\hline Jenny Zhan & ${ }^{1}$ Department of Pathology and ${ }^{2}$ ImmunoX, UCSF, San Francisco, California, USA. \\
\hline Bushra Samad & ${ }^{1}$ Department of Pathology and ${ }^{2}$ ImmunoX, UCSF San Francisco, California, USA. \\
\hline Suzanna Chak & $\begin{array}{l}{ }^{5} \text { Division of Pulmonary and Critical Care Medicine, Department of Medicine, UCSF, San } \\
\text { Francisco, California, USA. }\end{array}$ \\
\hline Rajani Ghale & $\begin{array}{l}5 \text { Division of Pulmonary and Critical Care Medicine, Department of Medicine, UCSF, San } \\
\text { Francisco, California, USA. }\end{array}$ \\
\hline Jeremy Giberson & $\begin{array}{l}{ }^{5} \text { Division of Pulmonary and Critical Care Medicine, Department of Medicine, Zuckerberg San } \\
\text { Francisco General Hospital and Trauma Center, UCSF, San Francisco, California, USA. }\end{array}$ \\
\hline Ana Gonzalez & $\begin{array}{l}{ }^{5} \text { Division of Pulmonary and Critical Care Medicine, Department of Medicine, Zuckerberg San } \\
\text { Francisco General Hospital and Trauma Center, UCSF, San Francisco, California, USA. }\end{array}$ \\
\hline Alejandra Jauregui & $\begin{array}{l}{ }^{5} \text { Division of Pulmonary and Critical Care Medicine, Department of Medicine, UCSF, San } \\
\text { Francisco, California, USA. }\end{array}$ \\
\hline Deanna Lee & $\begin{array}{l}{ }^{5} \text { Division of Pulmonary and Critical Care Medicine, Department of Medicine, Zuckerberg San } \\
\text { Francisco General Hospital and Trauma Center, Cardiovascular Research Institute, UCSF, San } \\
\text { Francisco, CA, USA. }\end{array}$ \\
\hline Viet Nguyen & $\begin{array}{l}{ }^{5} \text { Division of Pulmonary and Critical Care Medicine, Department of Medicine, Zuckerberg San } \\
\text { Francisco General Hospital and Trauma Center, Cardiovascular Research Institute, UCSF, San } \\
\text { Francisco, CA, USA. }\end{array}$ \\
\hline Kimberly Yee & $\begin{array}{l}{ }^{5} \text { Division of Pulmonary and Critical Care Medicine, Department of Medicine, University of } \\
\text { California San Francisco, Cardiovascular Research Institute, UCSF, San Francisco, CA, USA. }\end{array}$ \\
\hline Yumiko Abe-Jones & ${ }^{11}$ Division of Hospital Medicine, UCSF, San Francisco, California, USA. \\
\hline Logan Pierce & ${ }^{11}$ Division of Hospital Medicine, UCSF, San Francisco, California, USA. \\
\hline
\end{tabular}




\begin{tabular}{|c|c|}
\hline Name & Institution \\
\hline Priya Prasad & ${ }^{11}$ Division of Hospital Medicine, UCSF, San Francisco, California, USA. \\
\hline Pratik Sinha & $\begin{array}{l}{ }^{5} \text { Division of Pulmonary and Critical Care Medicine, Department of Medicine, UCSF, San } \\
\text { Francisco, California, USA. }\end{array}$ \\
\hline Alexander Beagle & ${ }^{5}$ Department of Medicine, UCSF San Francisco, California, USA \\
\hline Tasha Lea & ${ }^{1}$ Department of Pathology, UCSF San Francisco, California, USA. \\
\hline Armond Esmalii & ${ }^{12}$ Division of Hospital Medicine, University of California, San Francisco, CA, USA. \\
\hline Austin Sigman & $\begin{array}{l}{ }^{5} \text { Division of Pulmonary and Critical Care Medicine, Department of Medicine, University of } \\
\text { California San Francisco, San Francisco, California, USA. }\end{array}$ \\
\hline Gabriel M Ortiz & $\begin{array}{l}{ }^{11} \text { Department of Medicine, Zuckerberg San Francisco General Hospital and Trauma Center, } \\
\text { University of California San Francisco }\end{array}$ \\
\hline Kattie Raffel & ${ }^{12}$ Division of Hospital Medicine, University of California, San Francisco, CA, USA. \\
\hline Chayse Jones & $\begin{array}{l}{ }^{5} \text { Division of Pulmonary and Critical Care Medicine, Department of Medicine, University of } \\
\text { California San Francisco, San Francisco, California, USA. }\end{array}$ \\
\hline Kathleen Liu & $\begin{array}{l}{ }^{13} \text { Division of Nephrology, Department of Medicine, University of California at San Francisco } \\
\text { School of Medicine, San Francisco, CA, United States } \\
\text { Division of Critical Care Medicine, Department of Anesthesia, University of California at San } \\
\text { Francisco School of Medicine, San Francisco, CA, United States. }\end{array}$ \\
\hline Walter Eckalbar & $\begin{array}{l}{ }^{5} \text { Division of Pulmonary and Critical Care Medicine, Department of Medicine, Cardiovascular } \\
\text { Research Institute and CoLabs, UCSF, San Francisco, CA, USA. }\end{array}$ \\
\hline
\end{tabular}

\section{References}

1. Mathew D et al., Deep immune profiling of COVID-19 patients reveals distinct immunotypes with therapeutic implications. Science 369, (2020).

2. Schulte-Schrepping J et al. , Severe COVID-19 Is Marked by a Dysregulated Myeloid Cell Compartment. Cell 182, 1419-1440 e1423 (2020). [PubMed: 32810438]

3. Hadjadj $\mathrm{J}$ et al. , Impaired type I interferon activity and inflammatory responses in severe COVID-19 patients. Science 369, 718-724 (2020). [PubMed: 32661059]

4. Zilionis R et al., Single-Cell Transcriptomics of Human and Mouse Lung Cancers Reveals Conserved Myeloid Populations across Individuals and Species. Immunity 50, 1317-1334 e1310 (2019). [PubMed: 30979687]

5. Huang IC et al. , Distinct patterns of IFITM-mediated restriction of filoviruses, SARS coronavirus, and influenza A virus. PLoS Pathog 7, e1001258 (2011). [PubMed: 21253575]

6. Reyes M et al. , An immune-cell signature of bacterial sepsis. Nat Med 26, 333-340 (2020). [PubMed: 32066974]

7. Davizon-Castillo P, Rowley JW, Rondina MT, Megakaryocyte and Platelet Transcriptomics for Discoveries in Human Health and Disease. Arterioscler Thromb Vasc Biol 40, 1432-1440 (2020). [PubMed: 32295424]

8. Mason KD et al. , Programmed anuclear cell death delimits platelet life span. Cell 128, 1173-1186 (2007). [PubMed: 17382885]

9. Bongiovanni D et al. , Transcriptome Analysis of Reticulated Platelets Reveals a Prothrombotic Profile. Thrombosis and haemostasis 119, 1795-1806 (2019). [PubMed: 31473989]

10. Chen WS et al. , Uncovering axes of variation among single-cell cancer specimens. Nat Methods 17, 302-310 (2020). [PubMed: 31932777]

11. Pujadas E et al. , SARS-CoV-2 viral load predicts COVID-19 mortality. Lancet Respir Med 8, e70 (2020). [PubMed: 32771081]

12. Hue $\mathrm{S}$ et al. , Uncontrolled Innate and Impaired Adaptive Immune Responses in Patients with COVID-19 ARDS. Am J Respir Crit Care Med, (2020).

13. Wang $\mathrm{Y}$ et al. , Kinetics of viral load and antibody response in relation to COVID-19 severity. J Clin Invest 130, 5235-5244 (2020). [PubMed: 32634129] 
14. Bastard P et al. , Auto-antibodies against type I IFNs in patients with life-threatening COVID-19. Science, eabd4585 (2020). [PubMed: 32972996]

15. Michalska A, Blaszczyk K, Wesoly J, Bluyssen HAR, A Positive Feedback Amplifier Circuit That Regulates Interferon (IFN)-Stimulated Gene Expression and Controls Type I and Type II IFN Responses. Front Immunol 9, 1135 (2018). [PubMed: 29892288]

16. Dhodapkar KM et al. , Selective blockade of the inhibitory Fcgamma receptor (FcgammaRIIB) in human dendritic cells and monocytes induces a type I interferon response program. J Exp Med 204, 1359-1369 (2007). [PubMed: 17502666]

17. Bournazos S, Corti D, Virgin HW, Ravetch JV, Fc-optimized antibodies elicit CD8 immunity to viral respiratory infection. Nature, (2020).

18. Zuo Y et al. , Prothrombotic autoantibodies in serum from patients hospitalized with COVID-19. Science translational medicine 12, eabd3876 (2020). [PubMed: 33139519]

19. Consiglio CR et al. , The Immunology of Multisystem Inflammatory Syndrome in Children with COVID-19. Cell 183, 968-981 e967 (2020). [PubMed: 32966765]

20. Chakraborty S et al. , Proinflammatory IgG Fc structures in patients with severe COVID-19. Nat Immunol, (2020).

21. Huang H, Benoist C, Mathis D, Rituximab specifically depletes short-lived autoreactive plasma cells in a mouse model of inflammatory arthritis. Proc Natl Acad Sci U S A 107, 4658-4663 (2010). [PubMed: 20176942]

22. Looney RJ, Huggins J, Use of intravenous immunoglobulin G (IVIG). Best Pract Res Clin Haematol 19, 3-25 (2006). [PubMed: 16377538] 


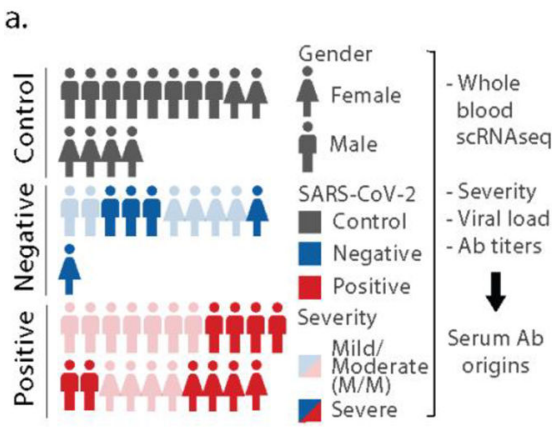

Neutrophils variants

C.

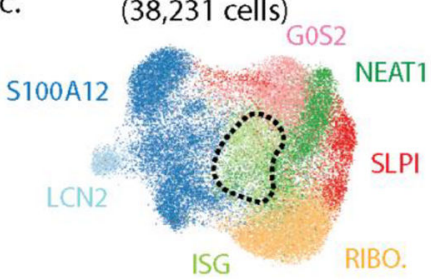

f.

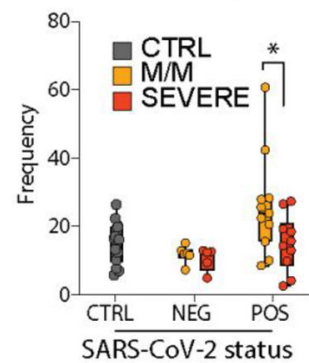

b. All Subjects: SARS-CoV-2 Positive and Negative

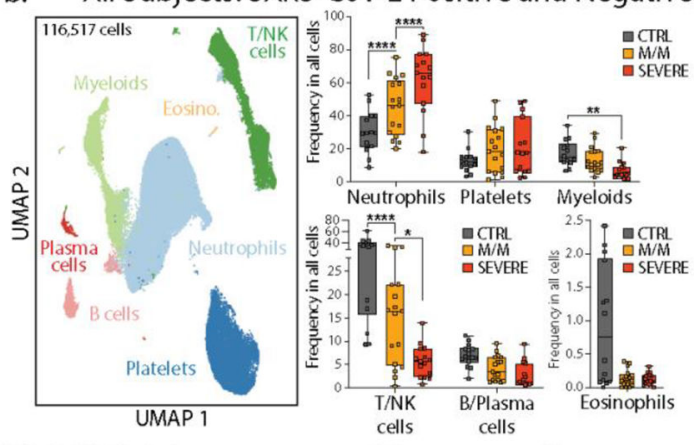

d. SARS-CoV-2 status

e. Disease severity
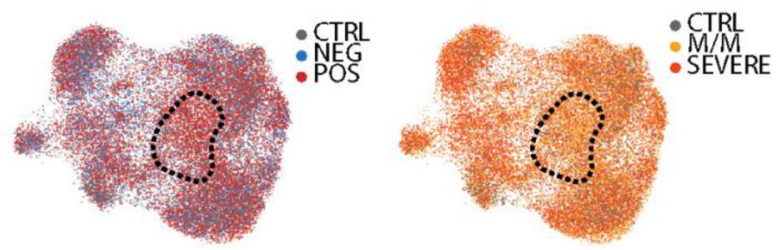

Figure 1: Severe COVID-19 disease is characterized by the lack of IFN-responsive neutrophils. a. Gender, SARS-CoV-2 status and disease severity in patients and control individuals (left) and description of study design (right) b. UMAP visualization of 116,517 cells merged from the entire cohort with specific populations overlaid (left), and frequencies of these populations across control, mild/moderate $(\mathrm{M} / \mathrm{M})$ and severe individuals (right). c. UMAP visualization of neutrophil subsets. d. and e. Overlay of SARS-CoV-2 status and disease severity, respectively, on the neutrophil UMAP. f. Frequency of ISG neutrophils among all neutrophils across SARS-CoV-2 status and disease severity (CTRL, n=14; NEG M/M, $n=6$; NEG severe, $n=5$; POS M/M, $n=11$, POS severe $n=10)$. g. Score of ISG signature across neutrophil subtypes and disease severity in SARS-CoV-2 positive patients. Statistical significance was assessed using a two-way ANOVA test with multiple comparisons for panel a and e, and using a two-sided Wilcoxon test for panel $\mathrm{f}$. $*$ p-value $<0.05$; ** p-value $<0.01$; $* * *$ p-value $<0.001 ; * * * *$ p-value $<0.0001$. Boxplot center, median; box limits, $25^{\text {th }}$ and $75^{\text {th }}$ percentile; whiskers, min. and max. data point. 
a.

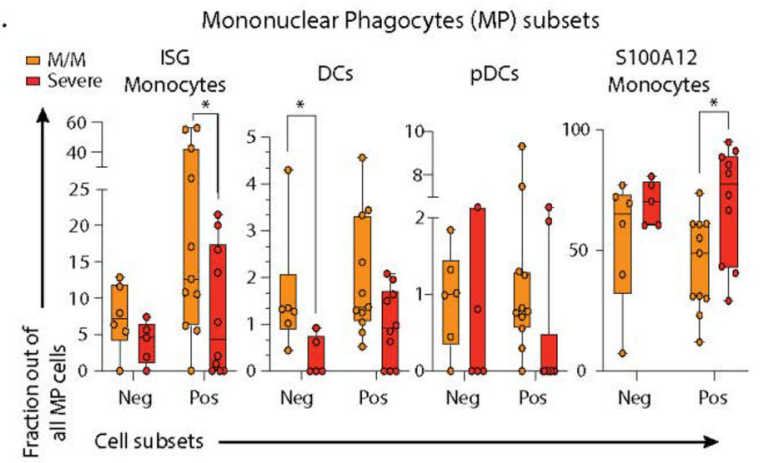

c. ISG score in T and B cells

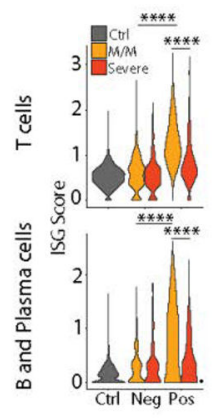

Global correlation between ISG populations

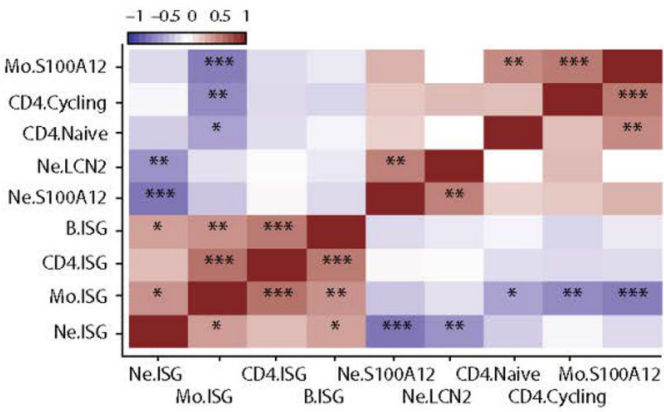

b. Correlation between ISG neutrophils and ISG monocytes

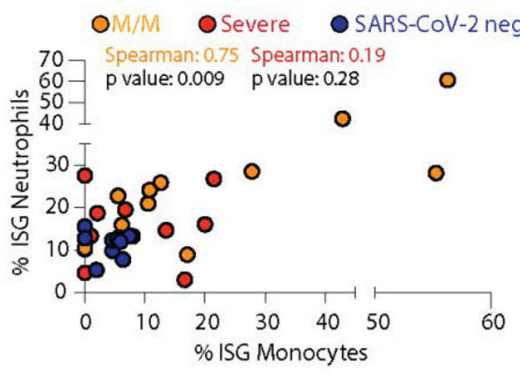

f. PhEMD analysis of all patients

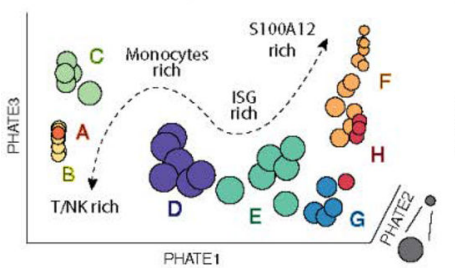

g. Overlay of SARS-CoV-2 status

h. Overlay of disease severity

e. ISG score in platelets

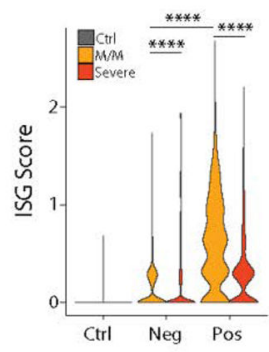

Figure 2: Severe COVID-19 disease is defined by the lack of a concerted IFN-response across peripheral blood immune cells.

a. Frequencies of MPC subsets among all MPC across mild/moderate (M/M, n=6 NEG, $\mathrm{n}=11$ POS) and severe ( $\mathrm{n}=5 \mathrm{NEG}, \mathrm{n}=10$ POS) individuals b. Scatter plot between neutrophil and monocyte ISG positive subsets patient by patient (M/M, $n=11$; severe, $n=10$; COVID-, $\mathrm{n}=11$ ). . Violin plot of ISG signature on all T cells (top) and all B/Plasma cells (bottom) across SARS-CoV-2 status and disease severity. Statistical significance was assessed using a two-sided Wilcoxon test. d. Correlation matrix using Spearman rank correlation between the most and the least correlated cell subsets to the Neutrophils ISG positive cells (data include all SARS-CoV-2 negative and positive patients). e. ISG signature score in all platelets across SARS-CoV-2 status and disease severity. f-h. 3D PhEMD embedding of all patients, colored by $\mathbf{f}$ de novo patient clusters A-H, g. SARS-CoV-2 status, and h. disease severity. Statistical significance was assessed using two-tailed Spearman's rank correlation (b) and Kruskal Wallis test with multiple comparisons (a), and two-sided Wilcoxon rank sum test for panels c and e. $*$ p-value $<0.05$; ** p-value $<0.01$; *** p-value $<0.001 ; * * * *$ p-value $<0.0001$. Boxplot center, median; box limits, $25^{\text {th }}$ and $75^{\text {th }}$ percentile; whiskers, min. and max. data point. 

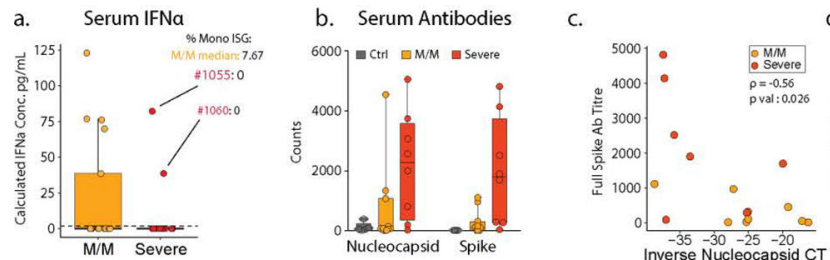

d.

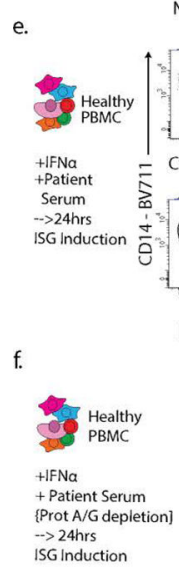

No Serum
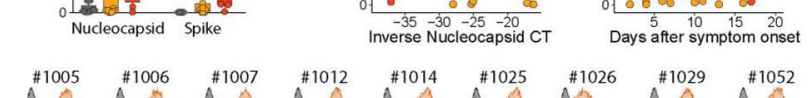

g.

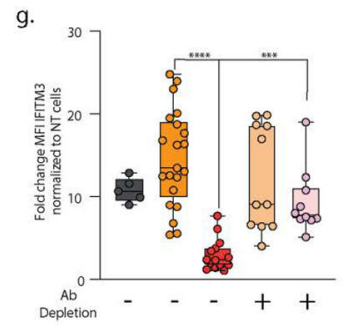

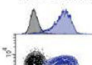

$\# 1005 \# 1006 \#$ \#1007 \#1012

Days after symptom onset
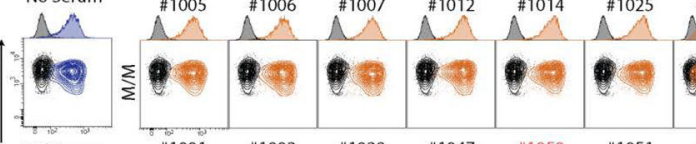

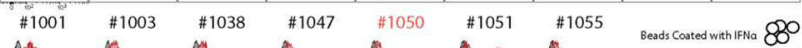

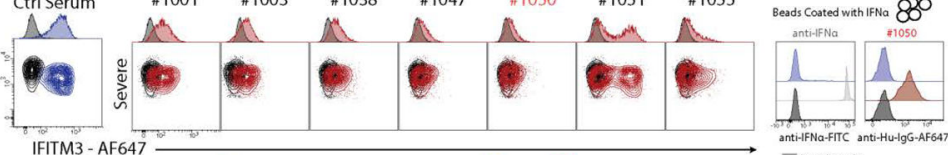

IFTM3 AF647 $\longrightarrow$

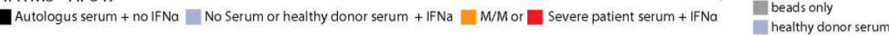

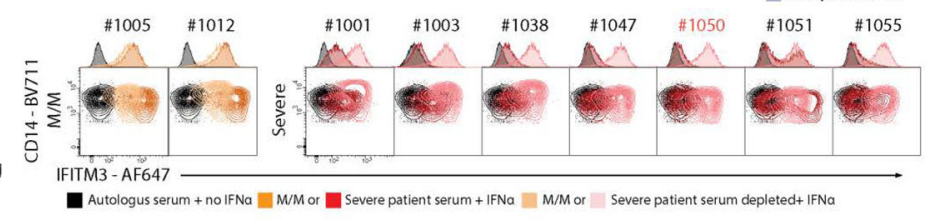

ECtrl Serum $\square$ M/M Serum $\square$ Severe Serum $\square$ M/M serum depleted $\square$ Severe serum depleted

h.
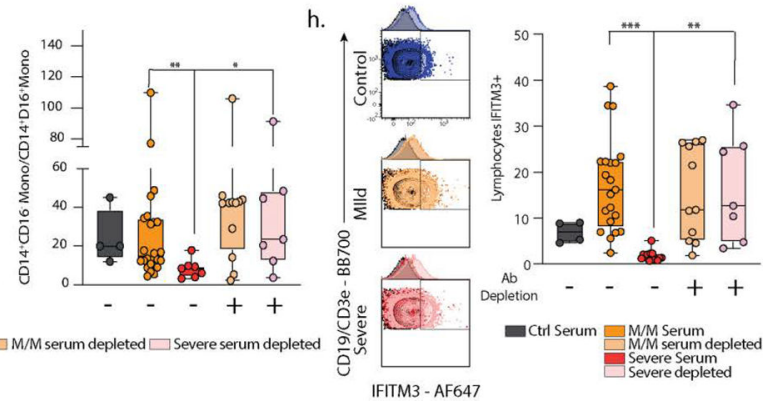

Figure 3: Neutralization of ISG induction by Antibodies from Severe COVID-19 Patients. a. Measurement of serum IFNa concentration from SARS-CoV-2 negative and positive M/M ( $n=17)$ or severe $(n=15)$ patients by ELISA. Patients 1055 and 1060 are highlighted in red and their Monocytes ISG frequency from Fig $2 \mathrm{C}$ is noted as well as the median for mild COVID-19 mild/moderate patients. Boxplot center, median; box limits, $25^{\text {th }}$ and $75^{\text {th }}$ percentile; whiskers, $1.5 \mathrm{x}$ interquartile range (IQR). b. Measurement of anti-SARSCoV-2 antibody levels in serum from patients by Luminex assay (M/M: Mild/Moderate). Boxplot center, median; box limits, $25^{\text {th }}$ and $75^{\text {th }}$ percentile; whiskers, min. and max. data point. c. Scatter plots showing viral load versus levels of antibody binding SARS-CoV-2 Nucleocapsid for patients in the cohort with severity overlaid. Antibody levels are shown as arbitrary units of MFI from Luminex assay while viral load is represented by an inverse CT number from QRT-PCR with target amplification of the SARS-CoV2 Nucleocapsid sequence. Correlation coefficient and significance calculated using Spearman's method. Patients for which data was unavailable were excluded (M/M, n=9; severe, $n=7$ patients) d. Scatterplot for SARS-CoV2 Full Spike protein antibody titers relative to days post symptom onset. Patients for which data was unavailable were excluded (M/M, $n=14$; severe, $n=8$ patients). e. Contour plots and histograms of CD14 and IFITM3 expression by monocytes from healthy PBMC cultured with IFNa and serum from either heathy donor, mild/moderate 
or severe SARS-CoV-2 positive patient. f. Contour plots and histograms of CD14 and IFITM3 expression by monocytes after pre-treating Mild/Moderate (light yellow) or Severe (pink) sera with protein $\mathrm{A} / \mathrm{G}$ prior to incubation with PBMC to deplete IgG. g. Boxplots of IFITM3 induction in CD14 monocytes (left; ctrl, n=5; M/M, n=21; severe, n=14; M/M depleted, $\mathrm{n}=11$; severe depleted, $\mathrm{n}=10$ ) and classical to intermediate monocytes ratio (right; ctrl, $\mathrm{n}=4 ; \mathrm{M} / \mathrm{M}, \mathrm{n}=24$; severe, $\mathrm{n}=7 ; \mathrm{M} / \mathrm{M}$ depleted, $\mathrm{n}=11$; severe depleted, $\mathrm{n}=7$ ) from 2 different experiment and 2 different healthy donors. h. Left: Contour plots and histograms of IFITM3 expression by pooled CD3+/CD19+ lymphocytes from healthy PBMC cultured with IFNa and serum from heathy donor, mild/moderate or severe SARS-CoV-2 positive patients. Light yellow and pink indicate respectively Mild/moderate and Severe sera pre-treated with protein A/G. Right: Box plot of IFITM3 induction in lymphocytes. Differences in g. and h. were calculated using a two-way ANOVA test with multiple comparisons. *p.value $<0.05$; $* *$ p.value $<0.01 ; * * *$ p.value $<0.001 ; * * *$ p.value $<0.0001$; ns: non-significant. For b/g/h boxplot center, median; box limits, $25^{\text {th }}$ and $75^{\text {th }}$ percentile; whiskers, min. and max. data point. 

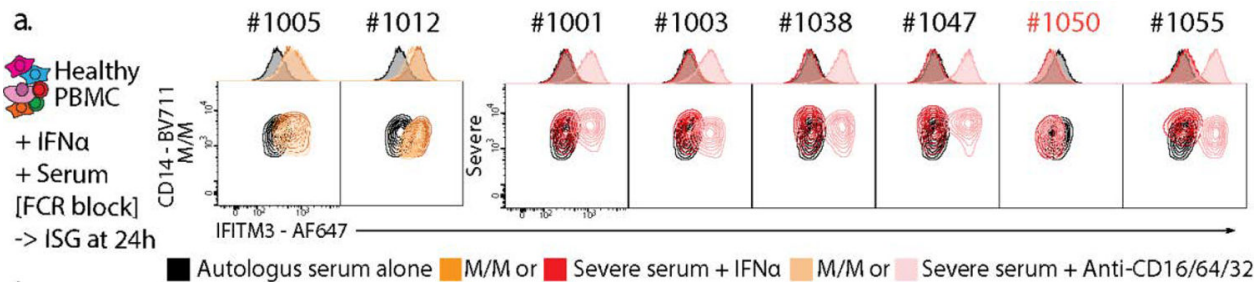

b.

Autologus serum alone $M / M$ or 1 severe serum +
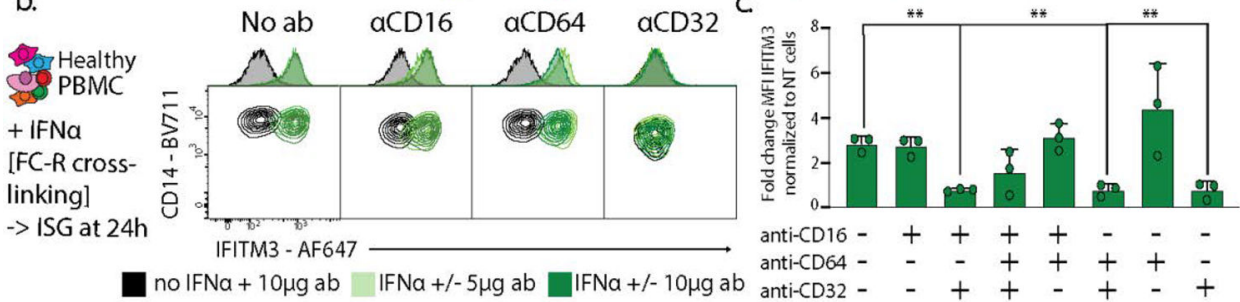

d.
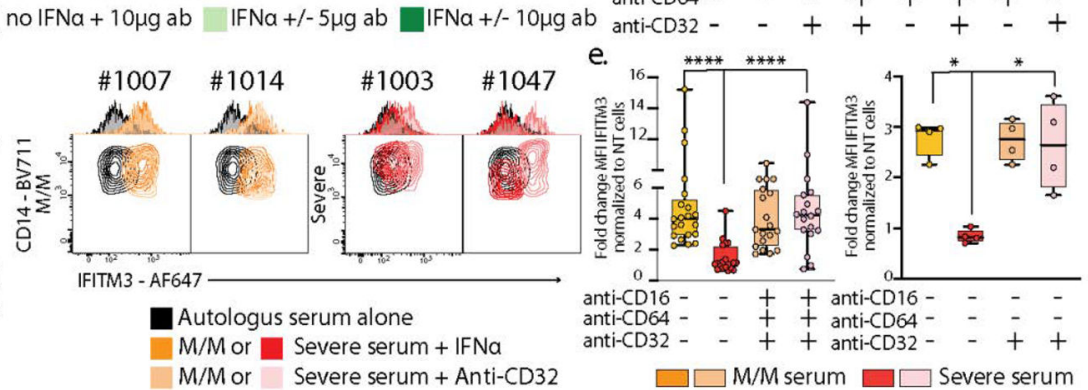

Figure 4: IgG-mediated neutralization of ISG induction by Severe COVID-19 Patients sera occurs through binding of their Fc to CD32.

a. Contour plots and histograms of CD14 and IFITM3 expression by monocytes from healthy PBMC cultured with IFNa and serum from either heathy donor, mild/moderate or severe SARS-CoV-2 positive patient, in the presence or not of anti-CD16/CD32/CD64 antibodies to block Fc receptors. b and c. CD14/IFITM3 contour plot and histograms (b) and boxplots presenting fold changes of IFITM3 expression (c) on CD14 monocytes after culturing healthy PBMCs $+/-$ IFNa $(1 \mathrm{pg} / \mu \mathrm{l})+/-5$ or $10 \mu \mathrm{g} / \mathrm{ml}$ of plate-coated isotype control, anti-CD16, anti-CD32 or anti-CD64 antibodies alone or in combination to crosslink and activate Fc receptors. Panel c presents the results of 2 independent experiments and 2 different cell donors, including two antibody quantities for one of the donors $(n=3$ experiments). Data is plotted as mean \pm SD. d. Neutralization assay as presented in panel a, with the sole addition of anti-CD32 blocking antibodies. e. Boxplots showing fold changes of IFITM3 expression for experiments presented in panel a (left graph, 5 independent experiments on 3 different cell donors) and panel d (right graph, 1 experiment on 2 different cell donors). Differences in c and e were calculated using a two-way ANOVA test with multiple comparisons. * p.value $<0.05$; ** p.value $<0.01$; **** p.value $<0.0001$. Boxplot center, median; box limits, $25^{\text {th }}$ and $75^{\text {th }}$ percentile; whiskers, min. and max. data point. * p.value $<0.05 ; * *$ p.value $<0.01 ; * * *$ p.value $<0.001 ; * * * *$ p.value $<0.0001$ 